\title{
Hyperparasitic spore-forming eukaryotes (Microsporidia, Haplosporidia, and Myxozoa) parasitizing trematodes (Platyhelminthes)
}

\author{
Yu.Ya. Sokolova ${ }^{1,2^{*}}$, R.M. Overstreet ${ }^{3}$ \\ ${ }^{I}$ Institute of Cytology, Russian Academy of Sciences, St. Petersburg, Russia, 4 Tikhoretski Av. St. \\ Petersburg, 194064 Russia. E-mail: yysokolova@gmail.com \\ ${ }^{2}$ School of Medicine and Health Sciences, George Washington University, 2300 Eye Str NW, 20037, \\ Washington DC, USA. \\ ${ }^{3}$ Gulf Coast Research Laboratory, University of Southern Mississippi, Ocean Springs, Mississippi, \\ USA.E-mail: robin.overstreet@usm.edu
}

ABSTRACT: Trematodes serve as outstanding hosts for a variety of parasites. This paper restricts those parasites to three groups of non-related taxa parasitizing trematodes and forming invasive spores similar in external morphology, which is why in the past these three groups of parasitic eukaryotes were united into the taxon "Sporozoa" (Kudo, 1924). (1) Trematode-infecting microsporidia (type Microsporidia Balbiani, 1882: Rozellomycota: Holomycota) are represented by more than 30 species. Among those, the species with sequenced barcode (SSU rDNA) are phylogenetically associated with either microsporidia from invertebrates, as the species of the genus Unikaryon (7 species), or fish microsporidia, as the species of the genera Pleistophora and Ovipleistophora or alike forms (4 species). However, most of the species that we place in the incertae cedis group, are known only by light-microscopy descriptions. Those were attributed by the authors to the genera Nosema (14 species) and Microsporidium (8 species), but in fact their taxonomic affiliation, phylogenetic position, and origin remain unknown. (2) Trematode-infecting haplosporidia (type Haplosporida Caulleri Mesnil, 1899: Ascetosporea: SAR) are represented by only one genus Urosporidium with a broad range of hosts among marine helminths and free-living invertebrates. The literature describes 10 Urosporidium spp. infecting trematodes. (3) Myxosporidia or Myxozoa (subclass Myxosporea Butchli, 1881: class Myxozoa Grasse, 1970: Cnidaria: Holozoa) infect mainly fish. All three species known from trematodes, belong to the genus Fabespora, and, likely, switched to hyperparasitism from fish. These three spore-forming groups demonstrate a diversity of characteristics and group-specific physiological mechanisms in addition to common adaptations responding to similar environmental conditions. These forms developed from genetically dissimilar material and exemplify an amazing power of convergent evolution, a phenomenon A.A. Dobrovolskii and other Russian scholars of parasitology demonstrated for a variety of parasitic groups. How to cite this article: Sokolova Yu.Ya., Overstreet R.M. 2020. Hyperparasitic sporeforming eukaryotes (Microsporidia, Haplosporidia, and Myxozoa) parasitizing trematodes (Platyhelminthes)// Invert. Zool. Vol.17. No.2.P.93-117. doi: 10.15298/invertzool.17.2.01

KEY WORDS: Convergent evolution, Trematoda, Haplosporidia, hyperparasitism, Microsporidia, Myxozoa, Nosema, Pleistophora, Unikaryon, Urosporidium.

Paper is dedicated to the memory of A.A. Dobrovolsky.

Статья посвящена памяти А.А. Добровольского. 


\title{
Гиперпаразитические спорообразующие эукариоты (Microsporidia, Haplosporidia и Myxozoa) - паразиты в трематод (Platyhelminthes)
}

\author{
Ю.Я. Соколова ${ }^{1,2}$, Р.М. Оверстрит ${ }^{3}$ \\ ${ }^{1}$ Институт цุитологии РАН, Тихореикий пр., д. 4, Санкт-Петербург, 194064, Россия. E-таil: \\ yysokolova@gmail.com \\ ${ }^{2}$ School of Medicine and Health Sciences, George Washington University, 2300 Eye Str NW, 20037, \\ Washington DC, USA. \\ ${ }^{3}$ Gulf Coast Research Laboratory, University of Southern Mississippi, Ocean Springs, Mississippi, \\ USA,E-mail: robin.overstreet@usm.edu
}

РЕЗЮМЕ: Трематоды хорошо известны как хозяева разнообразных паразитических организмов. В настоящей статье рассмотрены представителей трех неродственных таксонов эукариот, представители которых паразитируют в трематодах и формируют инвазионные споры, сходные по внешней морфологии, из-за чего в прошлом эти три группы паразитических эукариот объединялись в тип Sporozoa (Kudo, 1924). (1) Микроспоридии (тип Microsporidia Balbiani, 1882: Rozellomycota: Holomycota) представлены более чем 30 видами. Виды с отсеквенированным баркодом (участок МС рДНК) филогенетически связаны либо с микроспоридиями беспозвоночных, как виды рода Unikaryon (7 видов), либо с микроспоридиями рыб, как виды родов Pleistophora и Ovipleistophora (4 вида). Однакао большинство видов, которые мы поместили в группу incertae cedis, известны лишь по светооптическим описаниям и отнесены авторами описаний к родам Nosema spp. (14 видов) и Microsporidium spp. (8 видов). (2) Гаплоспоридии (тип Haplosporida Caulleri et Mesnil, 1899: Ascetosporea: Sar) представлены только одним родом Urosporidium, имеющим широкий спектр хозяев среди морских беспозвоночных, как паразитических, так и свободноживущих. В литературе описано10 видов, заражающих трематод. (3) Миксоспоридии или Myxozoa (п/класс Myхоsporea Butchli, 1881: класс Myхоzoa Grasse, 1970: тип Cnidaria: Holozoa), заражают, главным образом, рыб. Все 3 вида, известные из трематод, принадлежат к роду Fabespora, и, очевидно, перешли к гиперпаразитированию с рыб. Эти три генетически неродственные группы «Sporozoa» демонстрируют разнообразные признаки и физиологические механизмы, специфичные для каждой группы, но также и сходные адаптации к паразитизму, выраженные, в частности, в сходстве морфотипов и некоторых особенностей патогенеза. В целом, описанные группы эукариотических микроорганизмов представляют собой наглядный пример конвергентной эволюции - феномена, многократно описанного А.А. Добровольским и другими представителями Российской школы паразитологии на различных группах паразитов.

Как цитировать эту статью: Sokolova Yu.Ya., Overstreet R.M. 2020. Hyperparasitic spore-forming eukaryotes (Microsporidia, Haplosporidia, and Myxozoa) parasitizing trematodes (Platyhelminthes) // Invert. Zool. Vol.17. No.2. P.93-117. doi: 10.15298/ invertzool.17.2.01

КЛЮЧЕВЫЕ СЛОВА: конвергентная эволюция, Trematoda, Haplosporidia, гиперпаразитизм, Microsporidia, Myхоzoa, Nosema, Pleistophora, Unikaryon, Urosporidium. 


\section{Introduction}

This review is dedicated to Andrey Aleksandrovich Dobrovolskii, a brilliant university professor having a bright personality, a man of the highest culture and integrity, and an outstanding parasitologist specializing in trematodes. His fascinating lectures on protistology, invertebrate zoology, parasitology, and particularly on trematode life cycles, made listeners fall in love with the creatures that he discussed as well as with Nature and Science in general. These lectures inspired students of several generations, including the first author of this paper, to remain in the field of parasitology throughout their lives. This paper contains the overview of the current state of research on eukaryotic parasites of trematodes, thus covering subjects that always were in the focus on Prof. Dobrovolskii's interests, namely the biology of trematodes and the phenomenon of hyperparasitism. The fact that large parasites have smaller parasites and that they yet have smaller parasites has thrilled biologists for centuries, possibly before pointed out in an early poem (Swift, [1733] 1910). In conjunction with the microscope's invention, a mathematician (De Morgan, 1915) developed a rhyme based on Jonathan Swift's poem, "Big fleas have little fleas upon their backs to bite'em; and little fleas have lesser fleas, and so ad infinitum." The first comprehensive review of the known literature on hyperparasitic associations among helminths including parasites of trematodes, appeared in 1946 (Dollfus, 1946).

Trematodes seem to provide an acceptable home to a variety of eukaryotic parasites, like microsporidia, myxozoa, haplosporidia, ciliates, flagellates, and amoebae as well as bacteria and viruses. Complicated life cycles of trematodes that, in the course of their lifespans, infect several hosts belonging to various trophic levels, providing exceptional opportunities for the parasites to hitchhike between various invertebrates and vertebrates, particularly those in marine and freshwater ecosystems. Parasitizing trematodes should facilitate host-switching, spreading infections throughout the habitat, and horizontal gene transfer among secondary and primary hosts of the hyperparasites.

As an example, a parasite that we are not treating in detail (Overstreet, 1976b), we note a diplomonad flagellate (Hexamita sp.) in the intestine of Crassicutis archosargi (Digenea) but not in its fish host, the sheepshead (Archosargus probatocephalus), similar to a larger species of Hexamita in reproductive systems and eggs of Deropristis inflata (Digenea) in the American eel, Anguilla rostrata, from near Woods Hole, Massachusetts (Hunninen, Wichterman, 1938). Several authors (e.g., Dollfus, 1946; Overstreet, 1976b) discuss these other protists. A few trematodes of herbivorous hosts in Mississippi, such as the common mullet small haploporids ( $<1 \mathrm{~mm}$ long) Xiha fastigata (previously known as Dicrogasterf.) and Saccocoelioides beauforti (recently referred to as Culuwiya $b$.) contain motile bacteria in their excretory vesicles. A couple of attempts to extract the bacteria using capillary tubes to collect, culture, and identify (or sequence) were unsuccessful (RMO, unpublished data), but we encourage others to achieve this with the same or other hyperparasitic bacteria and techniques. The bacteria in the haploporids seem to be nonharmful symbionts unlike the bacterium $E d$ wardsiella ictaluri in the metacercaria of the diplostomoid Bolbophorus damnificus, which readily kills the hyper-host (commercial catfish, Ictalurus punctatus) and unlike other discussed associative bacteria (Overstreet, Lotz, 2016).

The purpose of this publication is to discuss the non-related spore-forming, eukaryotic parasites in trematodes, namely 1) Microsporidia (type Microsporidia Balbiani, 1882: Rozellomycota: Holomycota), 2) Haplosporidia (type Haplosporida Caulleri, Mesnil, 1899: Ascetosporea: Sar), and 3) Myxosporidia or Myxozoa (subclass Myxosporea Butchli, 1881: class Myxozoa Grasse, 1970: Cnidaria: Holozoa). In fact, the World Register of Marine species (WoRMS) places them in three kingdoms (Fungi, Phylum Microsporidia; Chromista, Phylum Cercozoa; and Animalia, Phylum Cnidaria). From recent phylogenies in the Tree of Life (Fig. 1) joining plants and animals, the first group (Microsporidia) shows a close relation- 


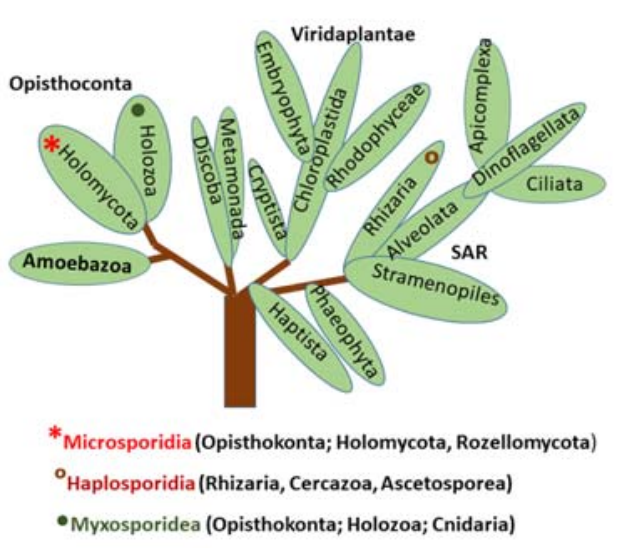

Fig. 1. Tree of Life (modified from Adl et al., 2019) demonstrates phylogenetic position of Microsporidia (Holomycota; Rozellamycota), Haplosporidia (SAR, Ascetosporea), and Myxosporidea (Holozoa, Cnidaria).

Рис. 1. Дерево Жизни (по Adl et al., 2019) демонстрирует филогенетическое положение Microsporidia (Holomycota; Rozellamycota), Haplosporidia (SAR, Ascetosporea) и Myxosporidea (Holozoa, Cnidaria).

ship to Fungi; the second, Haplosporidia, is associated with Foraminifera and brown algae; and the third, Myxosporidia represents highly reduced coelenterates (Adl et al., 2012, 2019). However, in early phylogenies, the orders Ascetosporea (including Haplosporidia), Microsporidia, and Myxozoa were united in the class "Sporozoa" (Kudo, 1924), not accepted nowadays. From the point of ecology, "Sporozoa" truly makes sense. The representatives of these groups share marine (or rarely freshwater) habitats, produce environmental spores that sometimes can hardly be distinguished from each other by the naked eye, infect the same host species, and often exhibit identical pathology. All together, these three major taxa reflect parallel evolutionary adaptations to the environment, which in the case of hyperparasites includes the habitat; primary (helminth) host; and the secondary host, or hyper-host (host of the helminth).

\section{Microsporidia}

The role of trematodes in host-switching and in overall shaping the evolutionary tree of Mi- crosporidia has yet to be established. Recent studies based on molecular data revealed a few peculiar phylogenetic associations of trematode-infecting microsporidia from crustacean and fish hosts with overlapping habitats, particularly marine and estuarine ones (Fig. 2). They suggest trophic transfer of microsporidian species, particularly those of the Clade 5 "Marinosporidia" (Vossbrinck et al., 2014; Stentiford et al., 2017a; Sokolova, Overstreet, 2018; Bojko et al., 2020). Presumably, some microsporidian taxa from the Clade 5 might have exploited life strategies of various parasites, including trematodes, to gain additional opportunities for transmission either within polyxenous life cycles or through predation of the infected host tissues by a potential alternative host (Stentiford et al., 2017a). However, direct evidence of a multihost invertebrate-fish parasite life cycle has been demonstrated only for Paranucleospora theridion, which uses a salmonid fish (Atlantic salmon, Salmo salar, or rainbow trout, Oncorhynchus mykiss) as an intermediate host and an ectoparasitic copepod (Lepeophtheirus salmonis or Caligus elongates) as a definite host (Freeman et al., 2003; Nylund et al., 2010; Freeman, Sommerville, 2011). We stress that hyperparasitism has been commonly reported among "higher" microsporidia (Phylum Microsporidia; Class Microsporea, sensu Sprague, 1977). Noteworthy that metchnikovellids (Phylum Microsporidia; Class Rudimicrosporea, Order Metchnikovellida sensu Sprague, 1977), the basal and diverged group of the phylum Microsporidia, are all gregarine-infecting hyperparasites (Sokolova et al., 2013, 2014). In addition to trematodes, hyperparasitic microsporidia also infect Myxozoa, both myxosporean developmental sequences from fish (Kudo, 1939; Diamant, Paperna, 1985) and actinosporian sequences from oligochaetes (Morris, Freeman, 2010); paramyxids (Stentiford et al., 2017b); cestodes (Dissanaike, 1957; Canning, Gunn, 1984; Poddubnaya et al., 2006); and parasitic copepods (Nylund et al., 2010; Freeman, Sommerville, 2011). Hitchhiking in other parasites, and particularly trematodes, may be considered as one potential route of transmission and radi- 


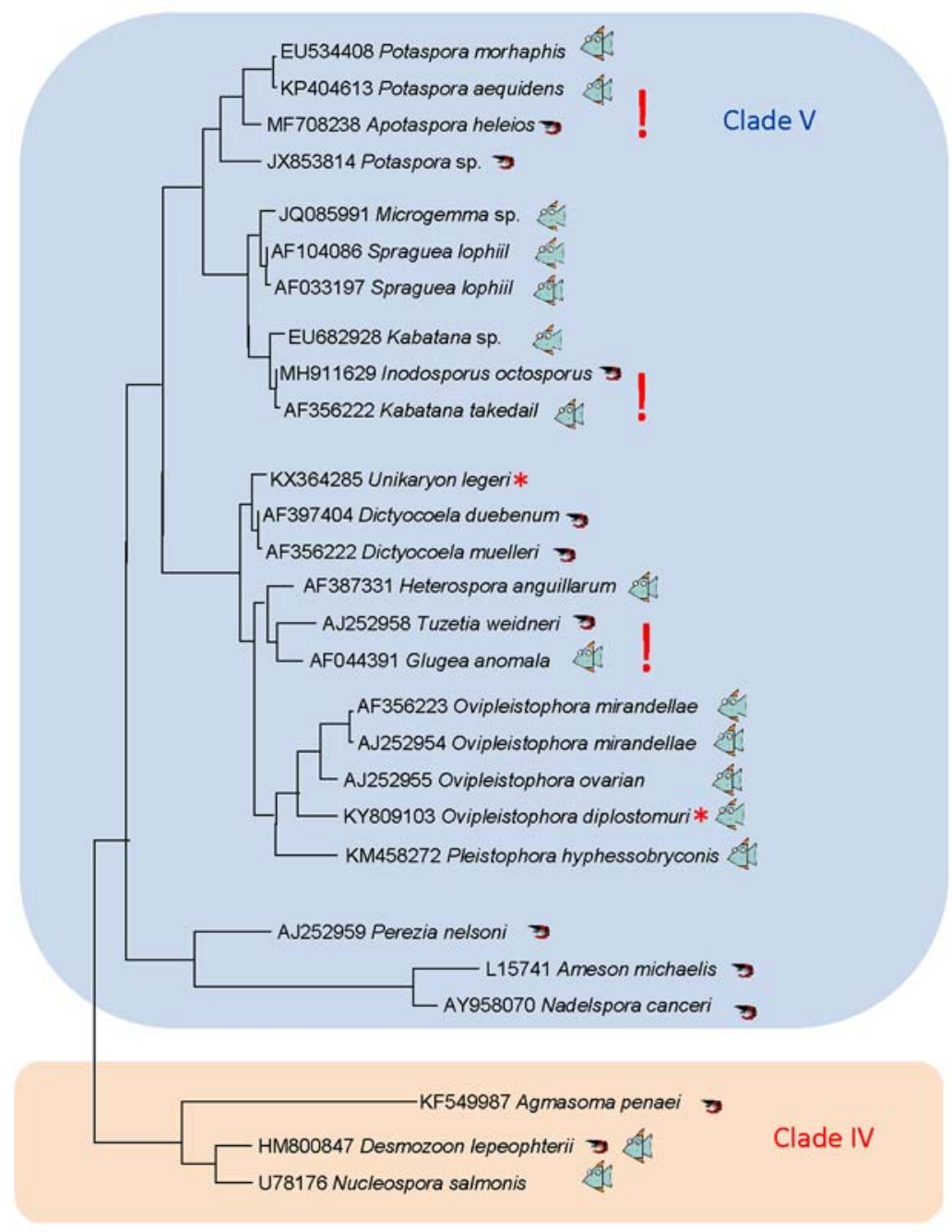

Fig. 2. Phylogenetic tree combined from several SSUrDNA-based analyses (Stentiford et al., 2017a; Lovy, Friend, 2017; Sokolova, Overstreet, 2018) of the fragment of the Clade V (Marinosporidia). This class contains mostly fish-infecting species (fish icons), but also several peculiar phylogenetic associations (exclamation marks) suggesting presumable role of hyper-parasites, like trematodes, in potential host switching between fish and crustacean hosts (shrimp icons). Species parasitizing trematodes, are indicated by asterisks. The outgroup Clade IV contains the only known microsporidium with a polyxenous fishcrustacean lifecycle (Desmozoon lepeophtherii).

Рис. 2. Филограмма, обобщающая несколько филогенетических анализов на основании МС рДНК (Stentiford et al., 2017a; Lovy, Friend, 2017; Sokolova, Overstreet, 2018), демонстрирует связи внутри Клады V (класс "Marinosporidia"). Эта клада состоит, в основном, из видов, заражающих рыб (изображение рыбы), но также содержит несколько странных филогенетических ассоциаций (восклицательные знаки), которые предполагают роль гиперпаразитов (возможно, трематод) в смене хозяев с рыб на ракообразных (изображение креветки) и обратно. Виды, паразитирующие у трематод помечены звездочками. Внешняя группа, Клада IV, содержит единственный вид микроспоридии (Desmozoon lepeophtherii) с подтвержденным поликсенным (рыба-ракообразное) жизненным циклом. 
ation of microsporidia (Stentiford et al., 2017a). Potential host-switching among infected trematode hosts or between trematodes and their hosts might have been facilitated by an exceptional ability of trematodes to suppress host innate immune responses. Mechanisms of this phenomenon, like suppression of macrophage activation through inhibiting Toll-like receptors (TLRs) and inhibition of inflammatory responses by parasite glycans, have been recently revealed and intensively studied (Mabbott, 2018). Thus infection with trematodes potentially could enable penetration of generally opportunistic microsporidia by creating immune-privelage niches.

To our knowledge, as many as 34 species of microsporidia have been reported from digenean hosts (Table 1), and this number continually grows: Victor Sprague in his comprehensive for the time "Annotated list of species" (Sprague, 1977) mentioned only 17 microsporidia infecting trematodes. Given the facts that the field has been understudied and most findings of microsporidia in trematodes are fortuitous, such a large number indicates that microsporidian infections in trematodes are fairly common in nature. Seven of the described species belong to the genus Unikaryon, four belong to the two closely-related genera of fish microsporidia, Pleistophora and Ovipleistophora, and the remaining 23 species occur distributed among the genera Nosema (14 species) and a collective taxon Microsporidium (8 species). However, we place them into an incertae sedis group, as they are described on the basis of light microscopic observations only that is not sufficient for discrimination. In contrast, electron microscopic observations exist for two of the four species of Pleistophora-Ovipleistophora and for all seven species of Unikaryon. Unfortunately, GenBank-accessible SSUrDNA sequences currently exist for only two species of microsporidia parasitizing trematodes (U. legeri, KX364285 and $O$. diplostomuri KY809102). Consequently, rDNA-based phylogenetic relationships of most species infecting trematodes remain obscure. Most described species are known from rediae and sporocysts developing in the first intermediate host, a mollusk, or in metacercariae developing in the second intermediate host which could be a mollusk, crustacean, or fish. Microsporidia and microsporidiainfected worms have never been isolated from birds, the definitive host of many marine species of trematodes (Shigina, 1986).

The genus Unikaryon Canning, Lai et Lie, 1974 with its type-species, U. pyriformis (see Canning et al., 1974), is composed of specialized parasites of helminths, mostly trematodes, but also cestodes (Sene et al., 1997). Currently, it includes two species of trematodes known from fishes and three species known from mollusks (Azevedo, Canning, 1987). In addition, two new yet undescribed species that share morphological similarity and $>95 \%$ of SSrDNA sequence identify with $U$. legeri, have been recently isolated from crab intermediate hosts (RMO, YYS, unpublished) (Table 1). All species of Unikaryon, except one, are known from larval stages, predominantly encysted metacercaria (see Table 1 for references). Probably, many species, infecting metacercariae in invertebrate hosts and currently attributed to Nosema (e.g., N. rhionica and N. strigeoideae) and to Microsporidium (e.g., M. spelotremae), belong to Unikaryon. The distinguishing morphological characters of Unikaryon spp. include the presence of prespore diplokaryotic stages in the life cycle, disporoblastic sporogony, and uninucleate spores with large posterior vacuoles. Spores are often arranged in pairs within a sporophorous vesicle membrane, which might be either robust or fragile, and occasionally reside in voluminous parasitophorous vacuoles individually or in pairs (Canning, Nicholas, 1974) (Fig. 3).

A few microsporidia infecting trematodes belong to the closely-related genera Pleistopho$r a$ and Ovipleistophora. Representatives of these genera predominantly infect adult trematodes parasitizing vertebrate hosts. Species in both genera produce multinucleate plasmodia transforming into sporophorous vesicles with multiple dimorphic micro- and macrospores (Table 1). These microsporidia seem to have switched to trematodes from fishes and potentially could 


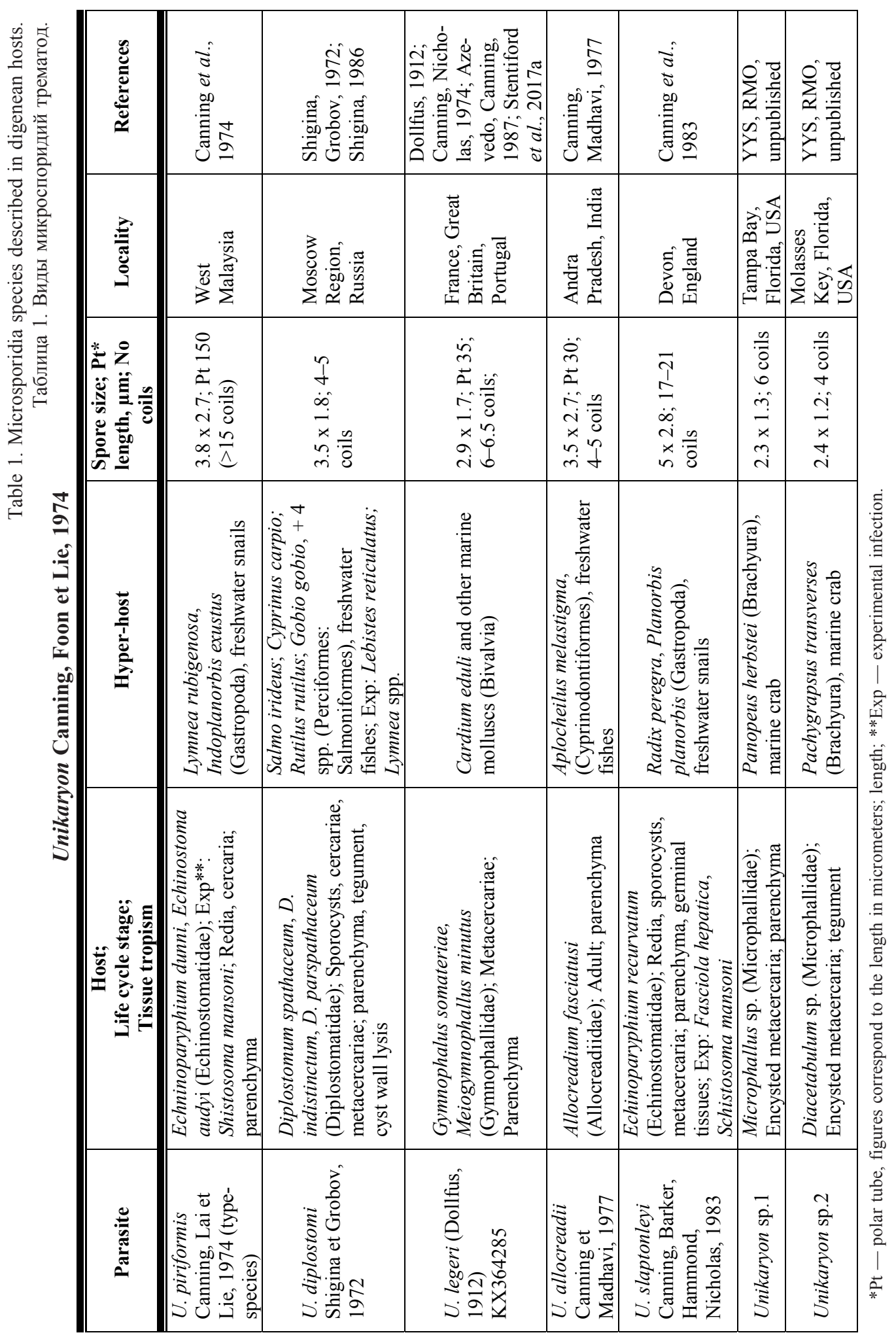




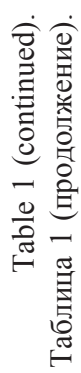

\begin{tabular}{|c|c|c|c|c|}
\hline 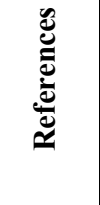 & 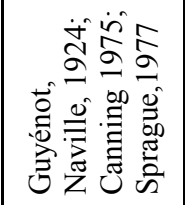 & 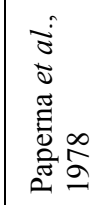 & 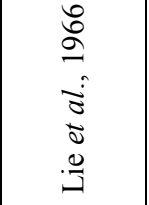 & 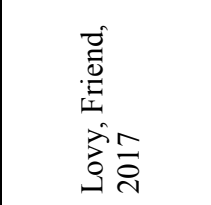 \\
\hline 氖 & 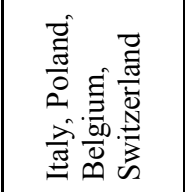 & 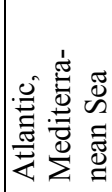 & 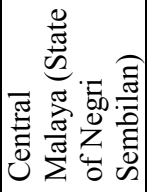 & 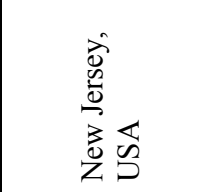 \\
\hline 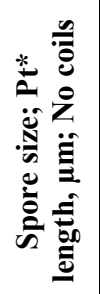 & 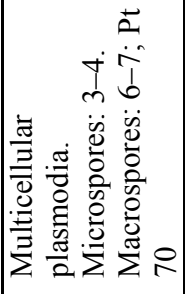 & 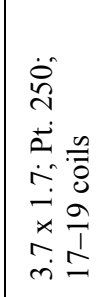 & 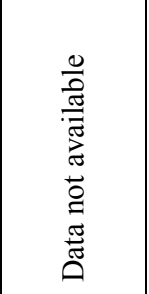 & 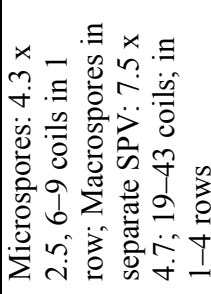 \\
\hline 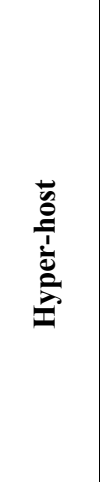 & 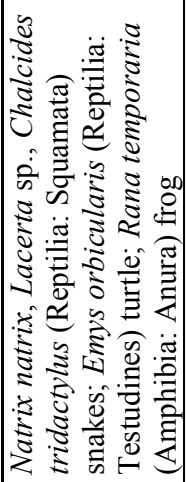 & 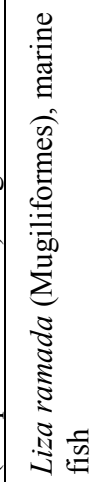 & 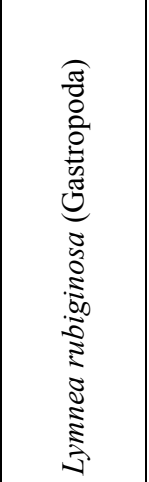 & 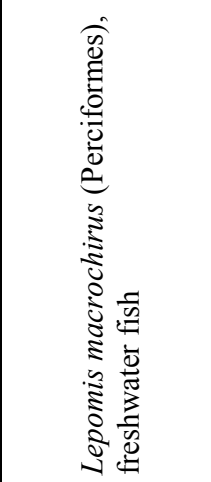 \\
\hline 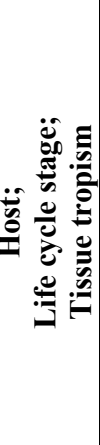 & 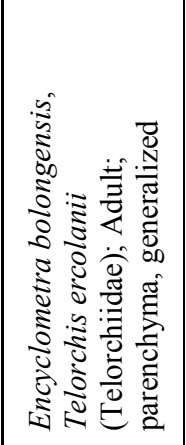 & 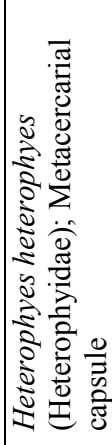 & 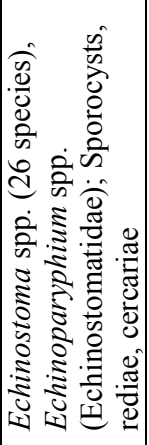 & 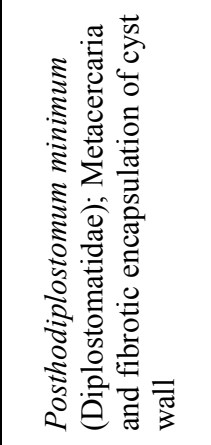 \\
\hline 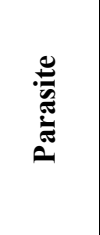 & 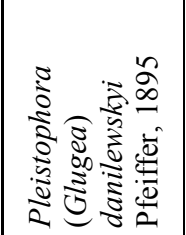 & 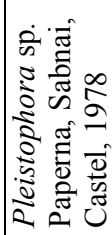 & 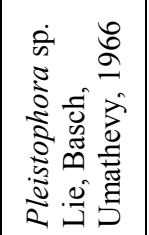 & 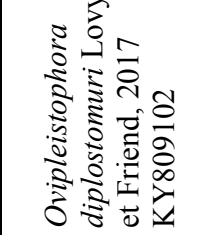 \\
\hline
\end{tabular}




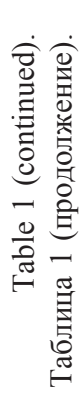

\begin{tabular}{|c|c|c|c|c|c|}
\hline 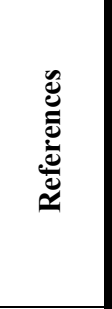 & 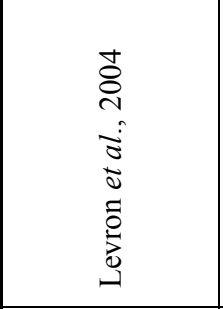 & 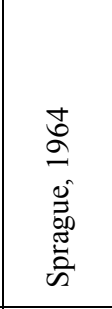 & 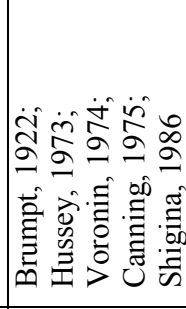 & 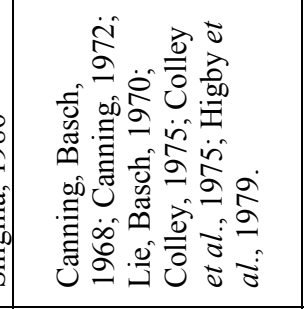 & 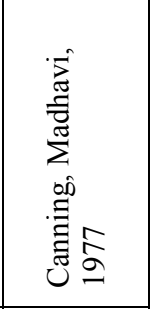 \\
\hline 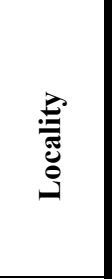 & 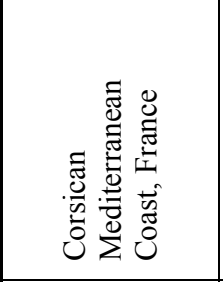 & 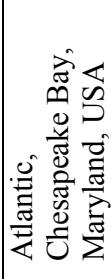 & 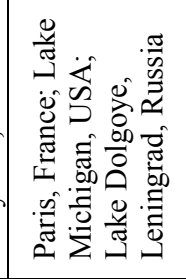 & 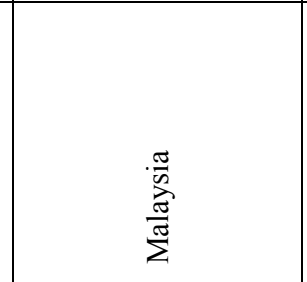 & $\stackrel{\pi}{\Xi}$ \\
\hline 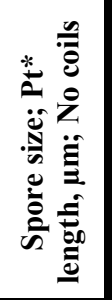 & $\begin{array}{l}\frac{n}{0} \\
0 \\
\tilde{b} \\
\ddot{+} \\
\dot{\vec{j}} \\
\dot{x} \\
\vec{i}\end{array}$ & 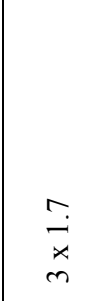 & $\begin{array}{l}\stackrel{\infty}{\sim} \\
\underset{\sim}{*} \\
\stackrel{\sim}{*}\end{array}$ & 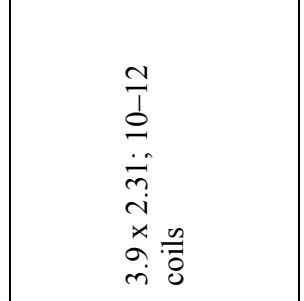 & 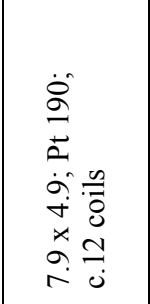 \\
\hline 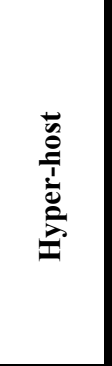 & 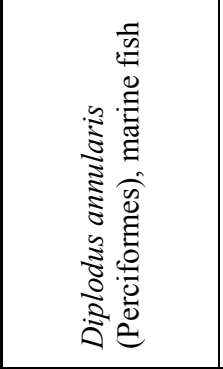 & 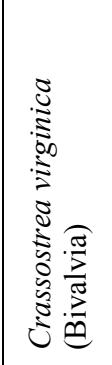 & 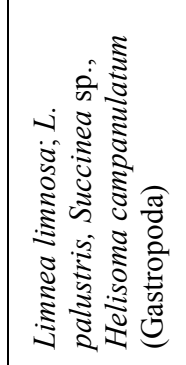 & 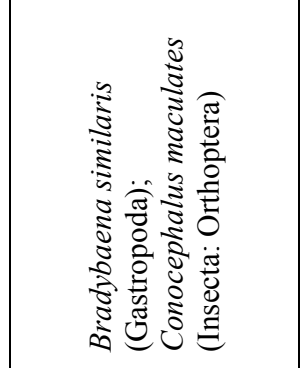 & 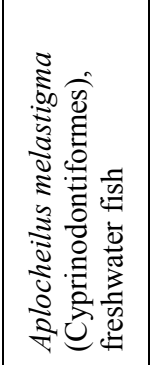 \\
\hline 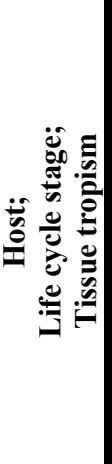 & 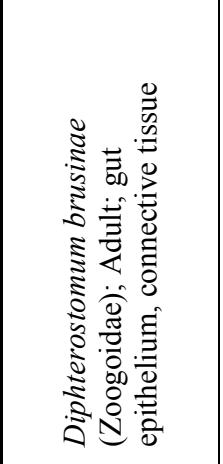 & 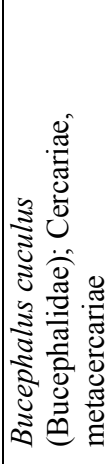 & 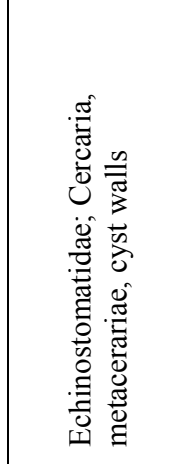 & 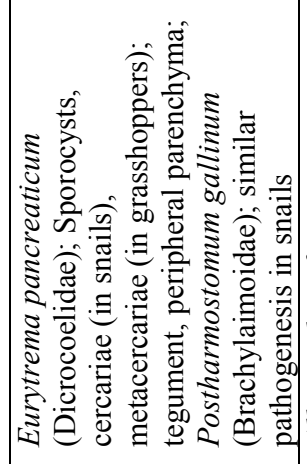 & 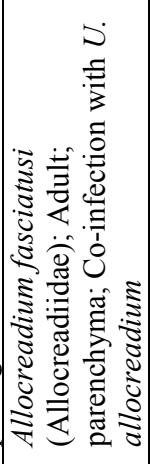 \\
\hline 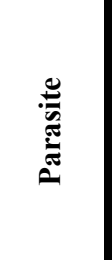 & 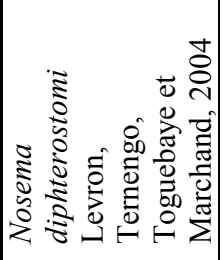 & 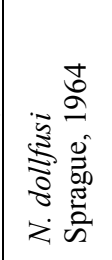 & 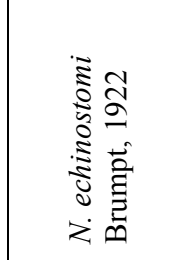 & 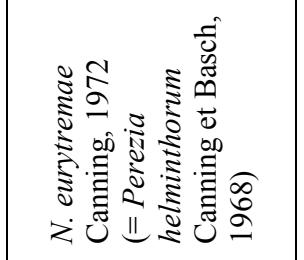 & 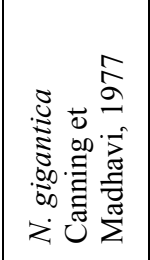 \\
\hline
\end{tabular}




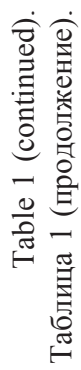

\begin{tabular}{|c|c|c|c|c|c|c|c|}
\hline 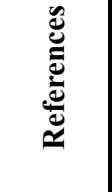 & 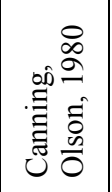 & 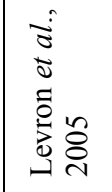 & 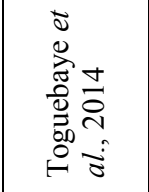 & 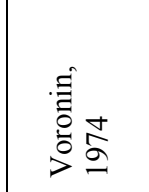 & 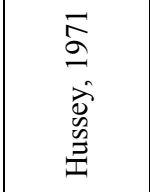 & 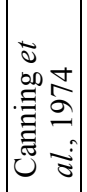 & 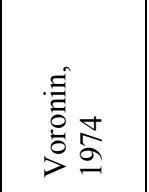 \\
\hline 胥 & 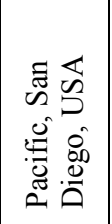 & 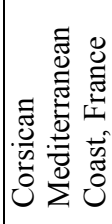 & 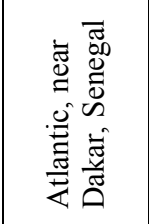 & 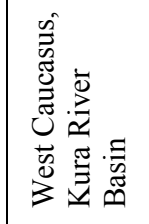 & 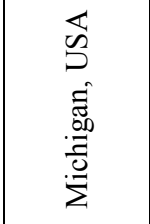 & 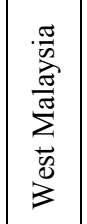 & 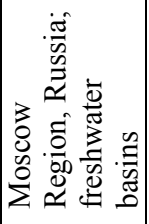 \\
\hline 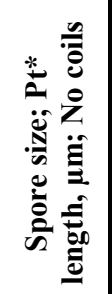 & $\begin{array}{l}\frac{n}{0} \\
0 \\
0 \\
\ddot{n} \\
\stackrel{n}{x} \\
\ddot{x} \\
\ddot{n}\end{array}$ & 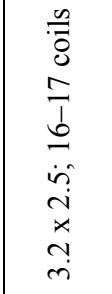 & $\begin{array}{l}0 \\
\overrightarrow{0} \\
0 \\
0 \\
I \\
= \\
= \\
\dot{0} \\
\dot{1} \\
\dot{x} \\
0 \\
\dot{m}\end{array}$ & 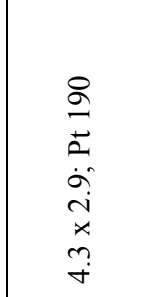 & $\begin{array}{l}8 \\
\varnothing \\
\Lambda \\
\dot{0} \\
\dot{0} \\
\dot{0} \\
\dot{x} \\
\dot{+}\end{array}$ & $\frac{\dot{b}}{\dot{x}}$ & $\begin{array}{l}\stackrel{m}{i} \\
x \\
\dot{v} \\
\dot{v}\end{array}$ \\
\hline 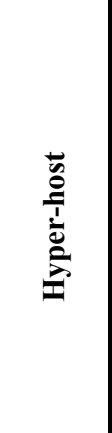 & 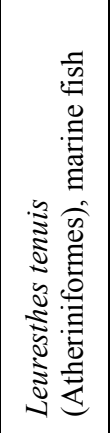 & 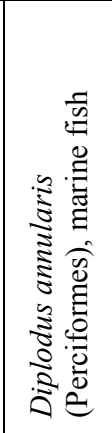 & 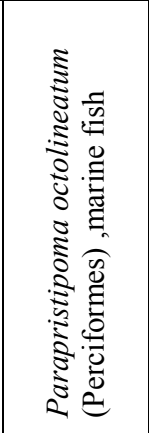 & 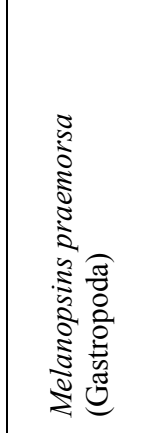 & 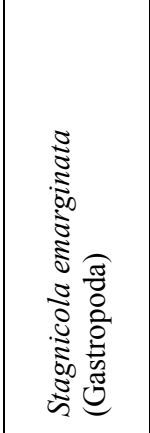 & 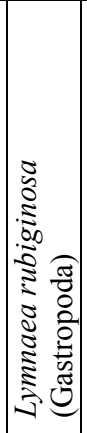 & 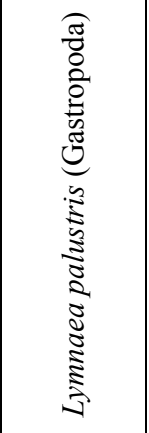 \\
\hline 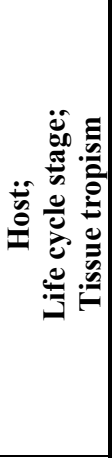 & 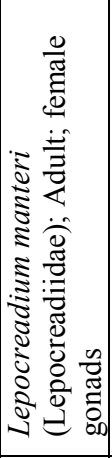 & 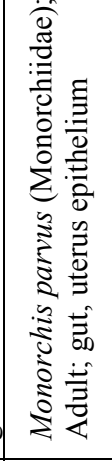 & 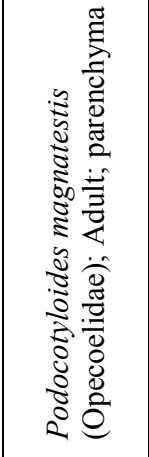 & 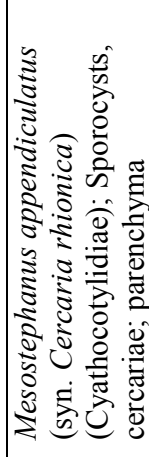 & 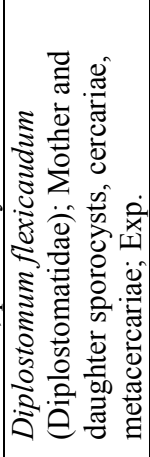 & 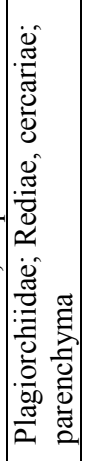 & 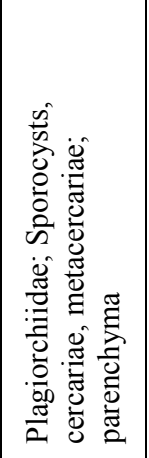 \\
\hline 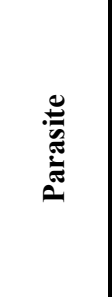 & 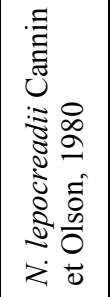 & 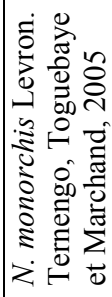 & 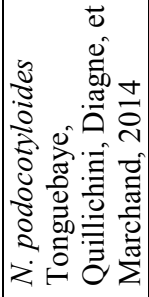 & 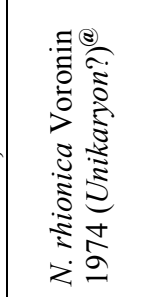 & 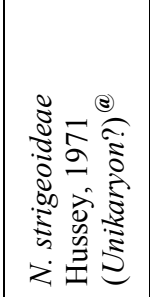 & 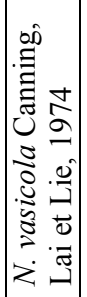 & 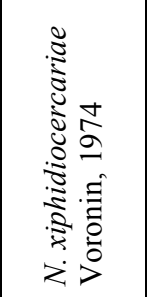 \\
\hline
\end{tabular}




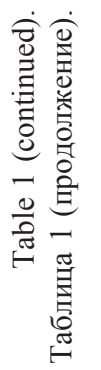

\begin{tabular}{|c|c|c|c|c|c|c|c|}
\hline 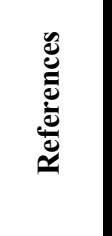 & 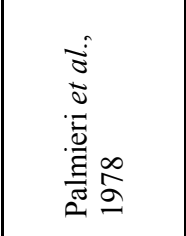 & 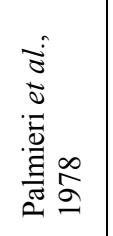 & 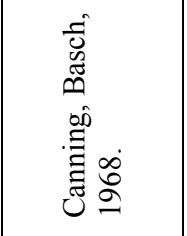 & 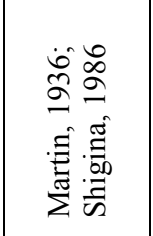 & 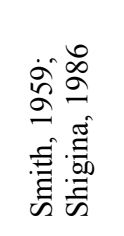 & 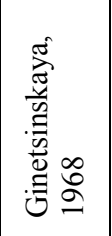 & 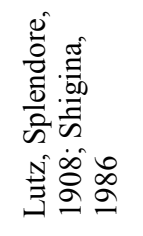 \\
\hline 苛 & 䏒 & $\underset{\mathscr{D}}{\overleftrightarrow{心}}$ & 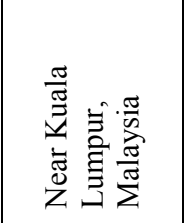 & 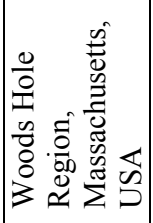 & 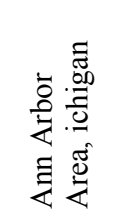 & 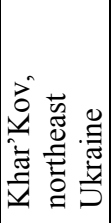 & $\begin{array}{l}\overline{\mathbb{N}} \\
\stackrel{\bar{n}}{n}\end{array}$ \\
\hline 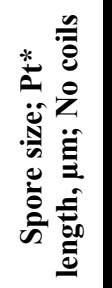 & 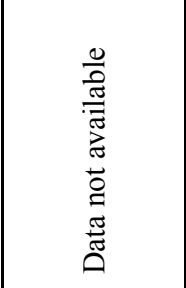 & $\begin{array}{l}\frac{0}{0} \\
\frac{\pi}{\pi} \\
\frac{\pi}{\pi} \\
\stackrel{0}{0} \\
\frac{\pi}{\pi} \\
0\end{array}$ & 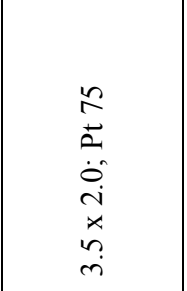 & 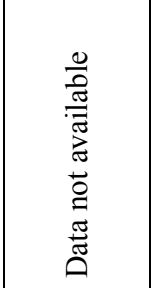 & 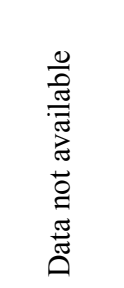 & 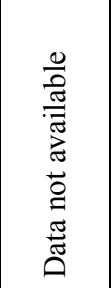 & 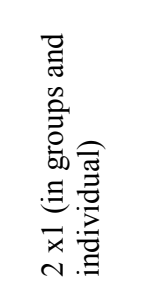 \\
\hline the & 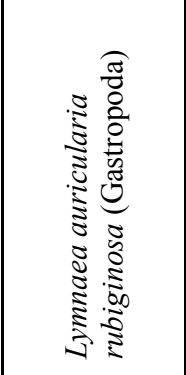 & 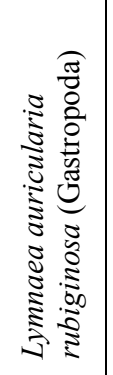 & 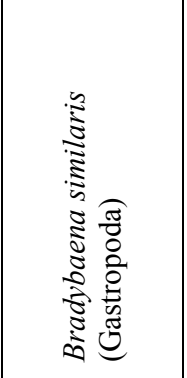 & 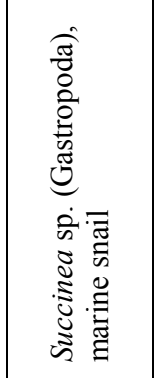 & 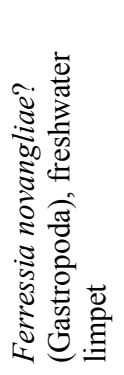 & 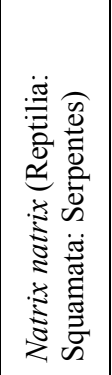 & 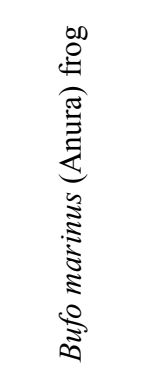 \\
\hline 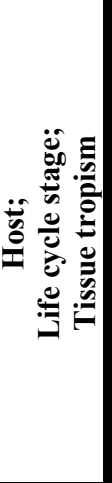 & 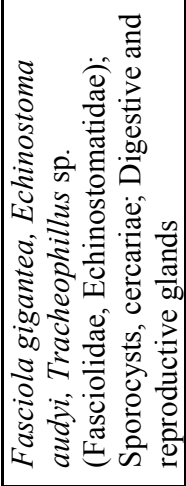 & 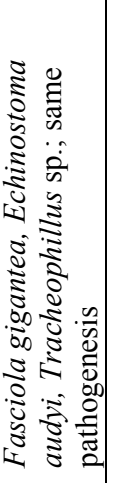 & 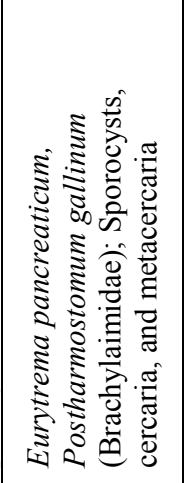 & 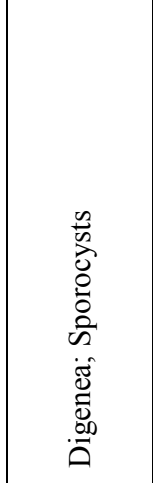 & 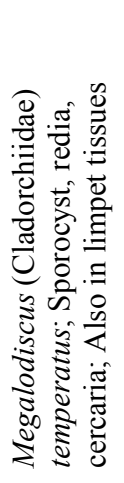 & 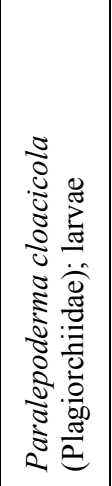 & 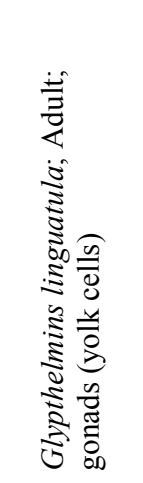 \\
\hline 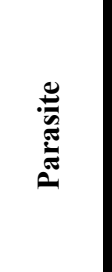 & 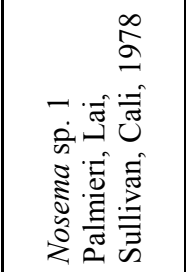 & 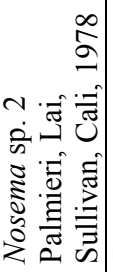 & 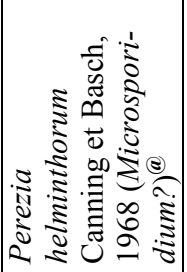 & 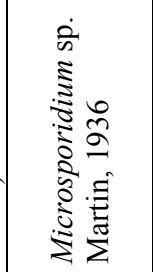 & 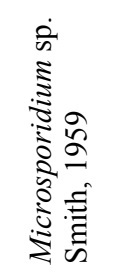 & 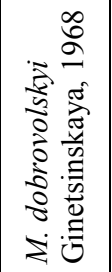 & 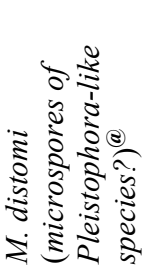 \\
\hline
\end{tabular}




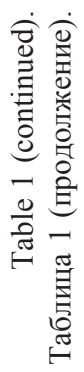

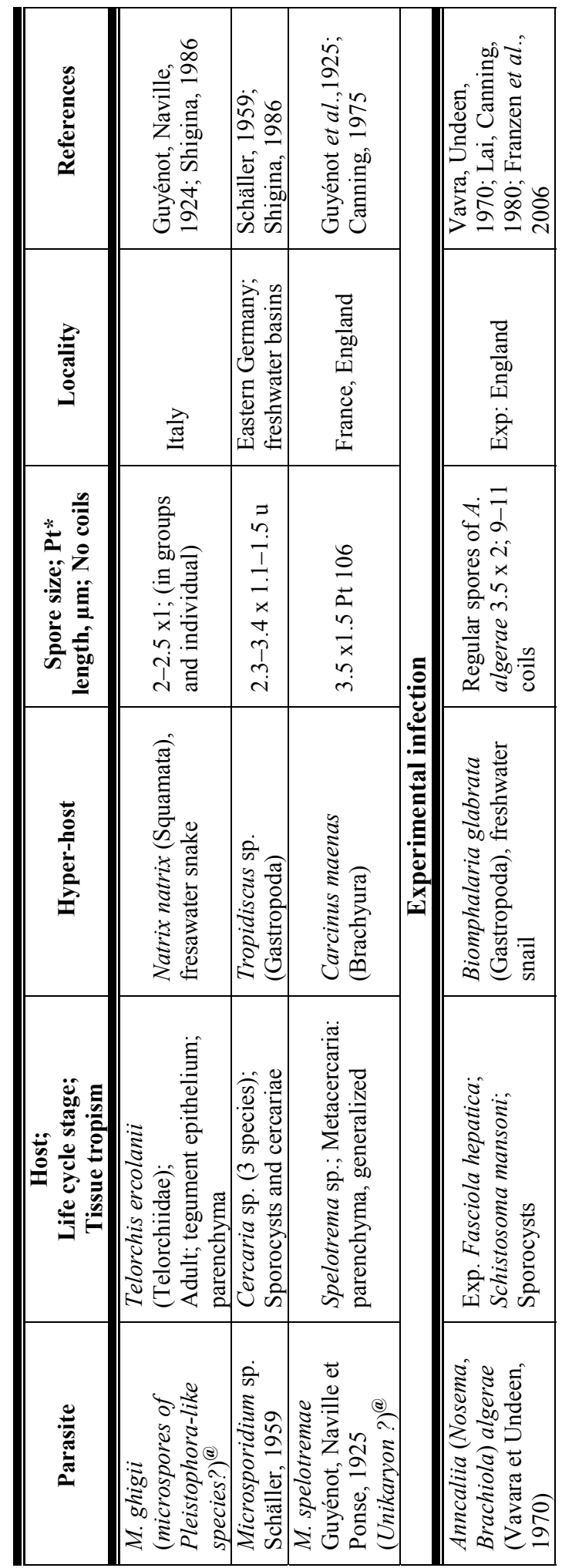



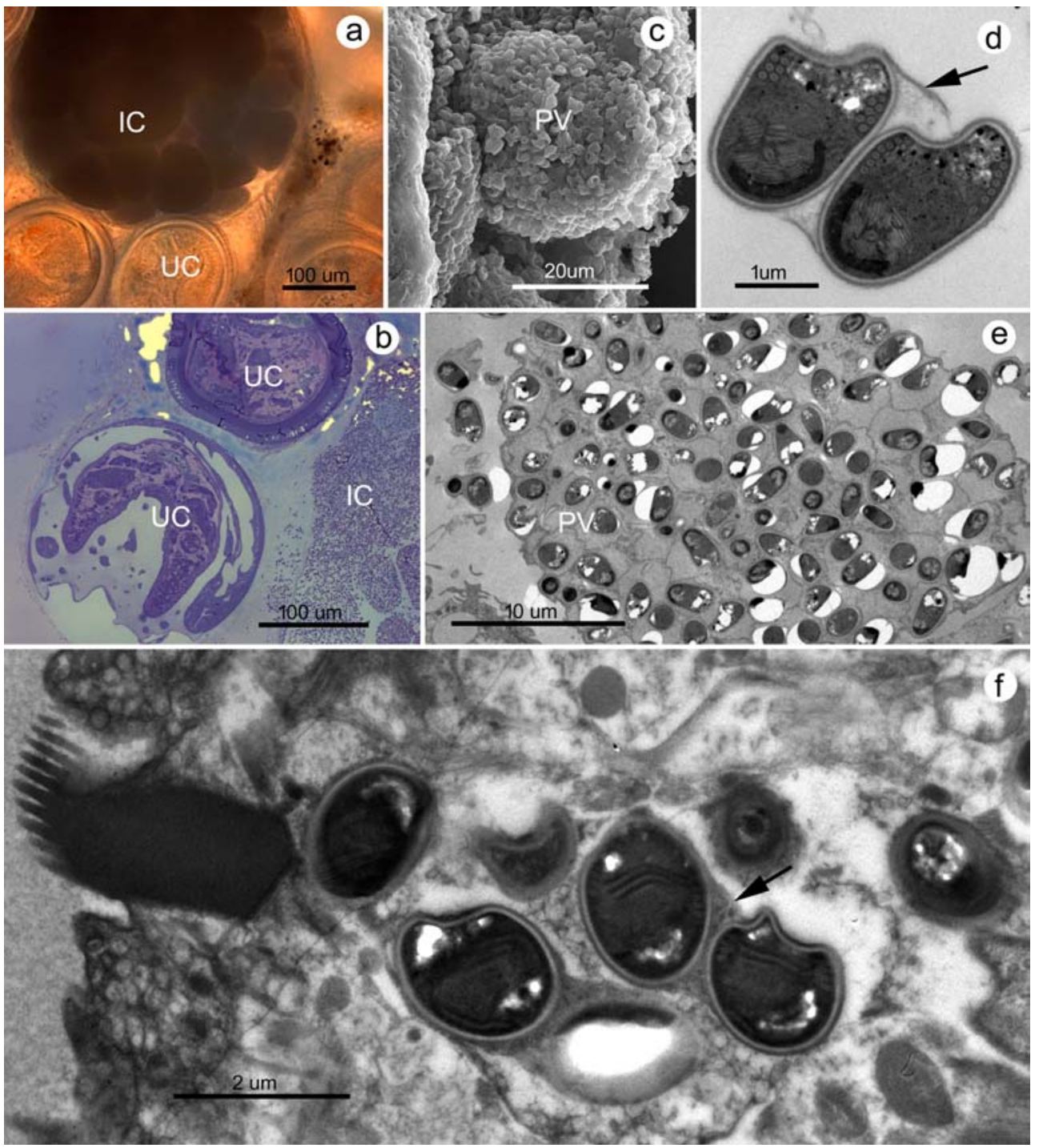

Fig. 3. Two new microsporidia of the genus Unikaryon isolated from trematodes infecting crabs: a-e Uniklaryon sp.1 from encysted metacercaria of Microphallus sp. in Panopeus herbstii from Tampa Bay, Florida, USA; f - Unikaryon sp. 2 from encysted metacercaria of Diacetabulum sp. in Pachygrapsus transversus from Molasses Key, Florida, USA. a - the infected metacercarian cyst (IC) is much larger than uninfected ones (UC). Unstained smear, light microscopy, DIC; b — infected (IC) and uninfected cysts (UC) on thick sections stained with methylene blue, bright field light microscopy; $\mathrm{c}$ - infected metacercaria in scanning electron microscope: the parasitophorous vacuole (PV) is tightly packed with spore duplets. $\mathrm{d}$ a duplet of spores enclosed in sporophorous vesicle membrane (arrow), at higher magnification, transmission electron viroscopy (TEM); e - parasitophorous vacuole (PV) filled with sporophorous vesicles, TEM; $\mathrm{f}$ Unikaryon sp. 2 exclusively infects submerged secretory epithelial cells in the trematode tegument. Arrow indicates the limits of the sporophorous vesicle that encloses two spores, TEM (membranes are poorly preserved because of initial fixation in paraformaldehyde).

Рис. 3 Два новых вида микроспоридий рода Unikaryon из трематод, заражающих крабов: a-e Uniklaryon sp.1 из инцистирующихся метацеркарий Microphallus sp. в Panopeus herbstii из Татра Bay, Florida, USA; f - Unikaryon sp. 2 из инцистирующихся метацеркарий Diacetabulum sp. в 
play a role in distribution of microsporidia among fishes, snakes, amphibians, and reptilians associated with an aquatic habitat and often hosting trematode parasites. For example, Pleistophora danilewskyi infects the trematode Telorchis ercolanii from the intestine of the grass snake, Natrix natrix, but also was recorded from snake tissues free of trematodes, as well as from lizards, turtles, and frogs that share habitats with the grass snake (Guyénot, Naville, 1924; Canning, 1975). One of four species identified as Pleistophora sp. was isolated from a gastropod and infected encysted metacercaria (Lie et al., 1966). Since ultrastructural studies were not conducted, and life cycle and morphological details were omitted from the original description, we strongly believe that this species was misidentified. Authors probably mistook parasitophorous vacuoles containing spores of $U n$ ikaryon, for sporophorous vesicles, characteristic for species of Pleistophora.

Pleistophora spp., as a rule, infect fish musculature (Sanders et al., 2010; Stentiford et al., 2013). In contrast, representatives of the closely-related genus Ovipleistophora that are morphologically and genetically similar to Pleistophora spp., infect oocytes, spermatocytes, and fibroblasts within ovaries and testes of perciform fishes (Summerfelt, Goodwin, 2010). The recently described Ovipleistophora diplostomuri infects fibroblasts, but only those that form fibroblastic capsules around metacercarial cyst walls of the diplostomoid trematode Posthodiplostomum minimum from internal organs of the bluegill sunfish, Lepomis macrochirus. $O$. diplostomuri clusters with trematodes infecting ovaries of perciform fish (Lovy, Friend, 2017). Tissue tropism similar to that of $O$. diplostomuri occurred in a microsporidium infecting fibro- blasts of metacercarial capsules of a heterophyid trematode (Heterophyes heterophyes) in the thinlip grey mullet, Chelon $(=$ Liza) ramada (Paperna et al., 1978). The authors assigned the microsporidium to Pleistophora sp., but more likely it is in fact conspecific with $O$. diplostomuri (Lovy, Friend, 2017). The unique tropism of Oviplestophora spp. may reflect the opportunistic nature of microsporidia. Oviplestophora mirandella, the type-species (Pekkarinen et al., 2002), infects fibroblasts in ovaries and testes that are known as immunologically privileged organs. O. diplostomuri, in turn, infects fibroblastic formations encapsulating the metacercarial cyst wall protected by trematode-induced immunosuppression. Hence, in some ways these two species share similar tissue tropism, both infecting fibroblasts in immunologically protected sites. $O$. diplostomuri intermediate hosts - trematodes Posthodiplostomum minimum and Heterophyes heterophyes parasitizing bluegills and mullets, respectively, produce long lasting infections in fish with $100 \%$ prevalence (Lovy, Friend, 2017). Long-term trematodefish associations might have helped creating specialized immunoprotective niches for microsporidia. Development of fish-related Pleistophora-like microsporidia in the metacercarial cyst-wall encapsulation composed of trematode secretion and fish host fibroblasts, exemplifies such a niche.

More than 20 species of trematode-infecting microsporidia have been placed in the genera Nosema and Microsporidium. According to the characteristics and host ranges provided by the authors, some of those species could be provisionally identified as Unikaryon spp. or Pleistophora spp. None of them has been sequenced, and hardly any of them appear to belong to the

Pachygrapsus transversus из Molasses Key, Florida, USA. а - циста зараженной метацеркарии (IC) намного больше чем незараженные метацеркарии (UC). Неокрашенный мазок, световая микроскопия, дифференциальный контраст по Номарскому; b — зараженные (IC) и незараженные цисты (UC) на полутонких срезах, окрашенных метиленовым синим; c - зараженные метацеркарии в сканирующий электронный микроскоп: паразитофорная вакуоль (PV) заполнена дуплетами спор; d сдвоенные споры, заключенные в мембрану спорофорного пузырька (стрелка), при большем увеличении, TEM; е - парзитофорная вакуоль (PV), заполненная спорофорными пузырьками, TEM; f Unikaryon sp. 2 заражает исключительно секректорные клетки погруженного эпителия тегумента. Стрелка указывает на границы спорофорного пузырька, в который заключены две споры, ТЕМ (мембраны сохранены плохо из-за первичной фиксации параформальдегидом). 
genus Nosema. We treat those species as an incertae sedis group (see Table 1 for the list and associated references).

A series of experimental studies on virulence, host range, and mass production of trematode-infecting microsporidia was driven by a potential ability of these hyperparasites to biologically control the pathogenic trematodes Fasciola hepatica and Schistosoma spp. that pose a serious threat to human health and cattle breeding worldwide. Suppressing trematode multiplication at parthenogenetic stages in molluscs by infecting the latter with microsporidia served as the major strategy for those experiments. Nosema eurytremae, with its unusually wide trematode host specificity, successfully propagated in lepidopteran larvae under experimental conditions (Higby et al., 1979; Pilley et al., 1978). Surprisingly, Anncaliia algerae was selected as the most preferred microsporidian species to be used in biological control (Lai, Canning, 1980). The latter species with dual natural host specificity in both mosquitoes and humans has unique generalist properties, allowing for experimental infections in a broad range of hosts and cell lines (Sokolova et al., 2019). Also, using N. strigeoideae against Diplostomum spatheceum infecting eye lenses of commercial and other fishes was explored (Palmieri et al., 1976). However, all these biological control studies using microsporidia in trematodes peaked in late 1970's - early 1980's (Shigina, 1986), did not have further development.

Overall, we conclude that microsporidia of trematodes are drastically understudied even though the complicated trematode polyxenous life cycles probably played an important role in dissemination of microsporidia among invertebrate and vertebrate hosts connected by trophic chains, especially in marine habitats with low density of inhabitants and limited transmission options.

\section{Haplosporidia}

All the described Ascetosporea haplosporidians infecting trematodes belong to the genus Urosporidium Caullery, Mesnil, 1905. The tax- on seems small, including four species by WoRMS and nine species in a recent review (Azevedo, Hine, 2017). By examining Table 2, one can see there are at least 14 species, including 4 unnamed ones, and probably several more exist.

Most species of Urosporidium infect trematodes, and those that do not still infect worms: turbellarian, cestode, nematode, and even a polychaete (the type species). Seven different families of trematodes have been reported as hosts, which indicates a wide range of hosts, especially when considering the other listed worm hosts (Table 2). Molecular phylogenies show haplosporidians are cercozoans related to Foraminifera and Radiolaria and probably date back to the Cambrian (Azevedo, Hine, 2017). Strict jackknife consensus tree of parsimony analysis of SSU rDNA sequence data of two species of Urosporidium show them to be sister to the other known haplosporidian genera (Burreson, Ford, 2004), but maximum likelihood trees show it to be nested among species of Haplosporidi$u m$; however, this tree remains unresolved (Azevedo, Hine, 2017).

Microscopy shows species of Urosporidium to have an internal flap of wall material covering the spore orifice as opposed to an external hinged lid in those of Haplosporidium and Minchinia, none of which infects trematodes (Azevedo, Hine, 2017). The flask-shaped spores of most species have one or more extensions, commonly referred to as tails (Table 2), and their number and length are not consistent; the size of spores does not necessarily present comparable values because some preparations are fixed, and others are fresh, under intense pressure, in sectioned material, or in ultrastructural images. Consequently, values should be used as a guide only and not as a taxonomic character. As the listed and additional species are examined more critically, including investigation by molecular techniques, changes in the characteristics of Urosporidium, other haplosporidian genera, and associated nomenclature will be made. For example, a few species of Haplosporidium are reported from worms, such as Haplosporidium malacobdellae in the nemerte- 


\begin{tabular}{|c|c|c|c|c|c|c|c|c|}
\hline 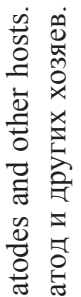 & 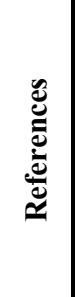 & 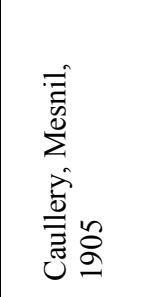 & 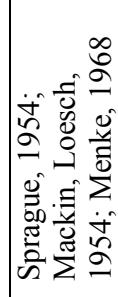 & 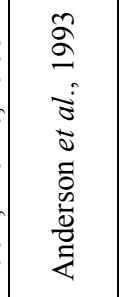 & 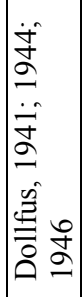 & $\begin{array}{l}\hat{0} \\
\circ \\
=0 \\
\overline{0} \\
0 \\
0\end{array}$ & 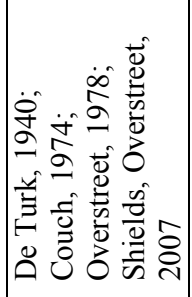 & 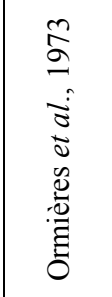 \\
\hline 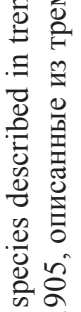 & 氖 & 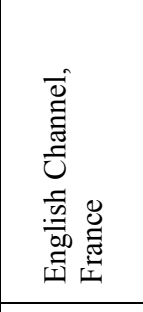 & 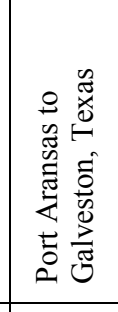 & 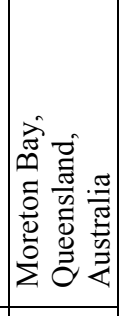 & 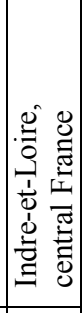 & 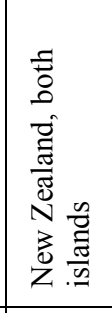 & 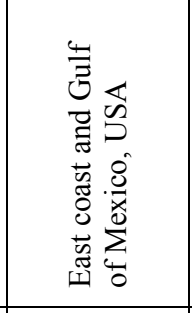 & 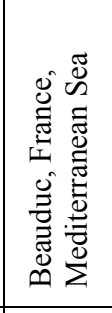 \\
\hline 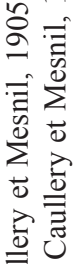 & 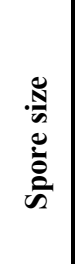 & 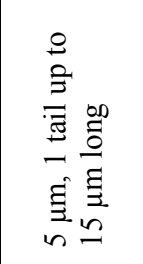 & 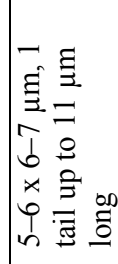 & 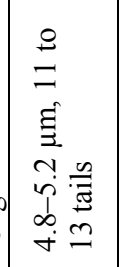 & 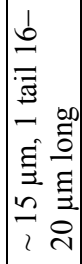 & 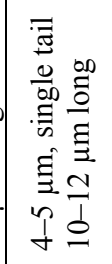 & 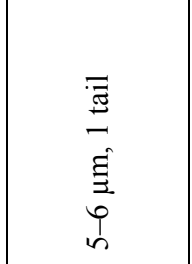 & 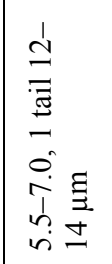 \\
\hline 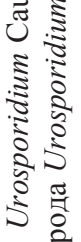 & 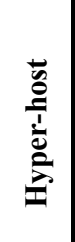 & & 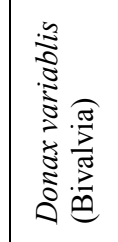 & 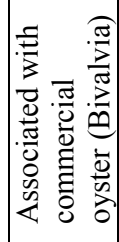 & 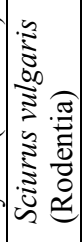 & 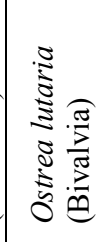 & 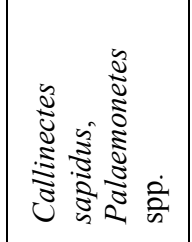 & 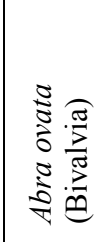 \\
\hline 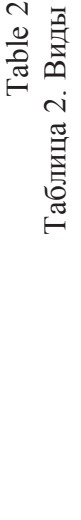 & 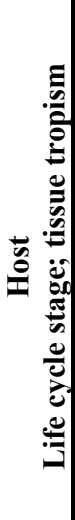 & 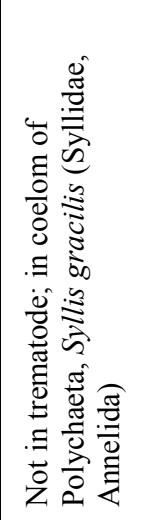 & 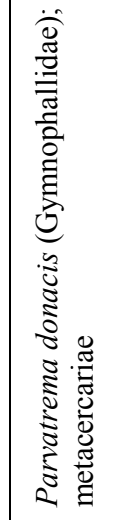 & 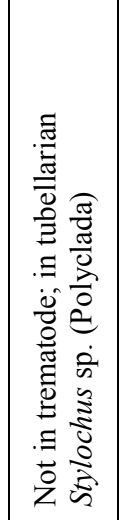 & 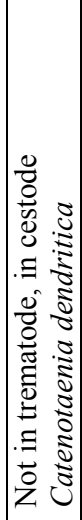 & 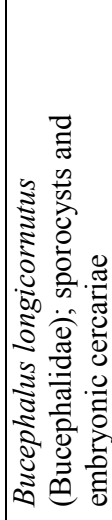 & 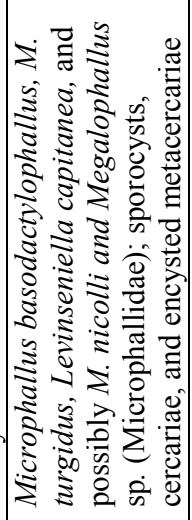 & 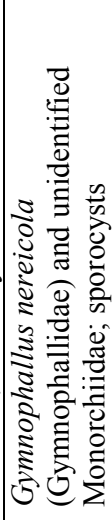 \\
\hline & 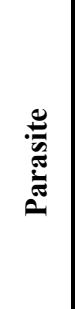 & 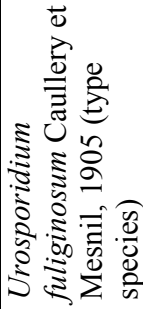 & 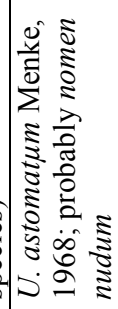 & 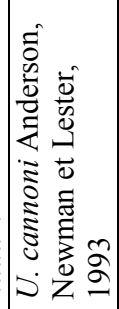 & 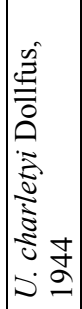 & 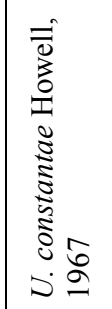 & 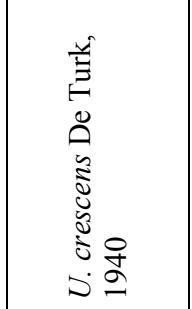 & 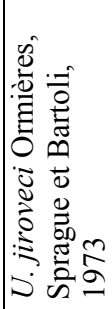 \\
\hline
\end{tabular}




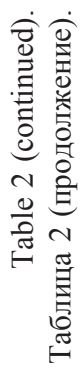

\begin{tabular}{|c|c|c|c|c|c|c|c|c|}
\hline 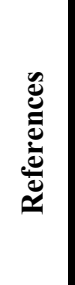 & 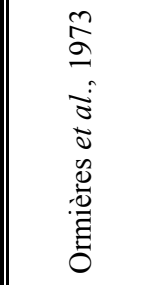 & 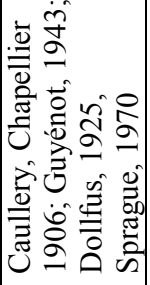 & 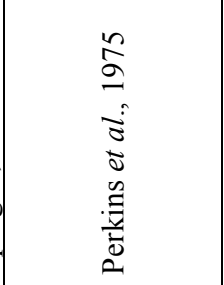 & 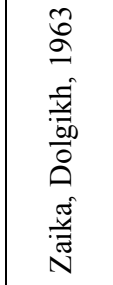 & 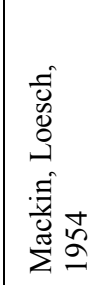 & 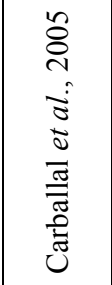 & 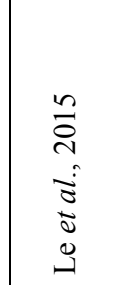 & 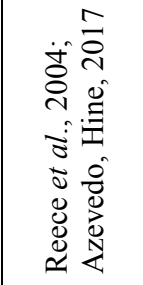 \\
\hline 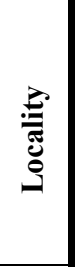 & 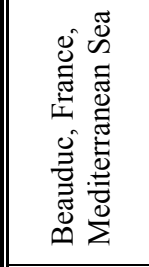 & 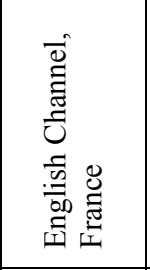 & 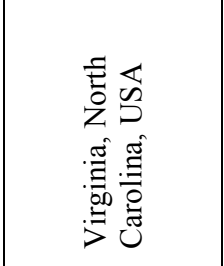 & 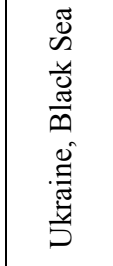 & 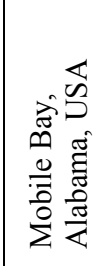 & 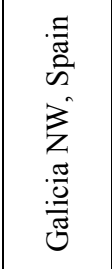 & 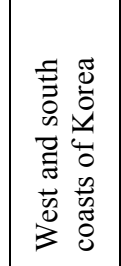 & 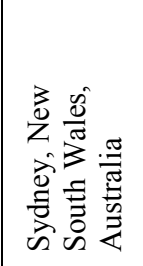 \\
\hline 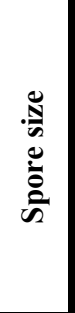 & 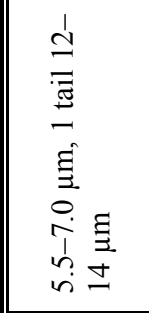 & 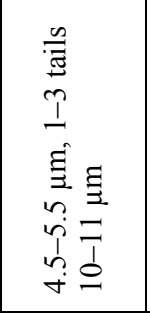 & 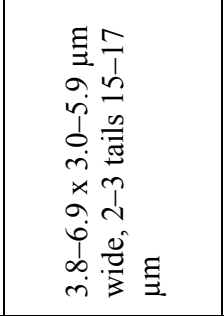 & 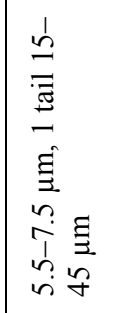 & 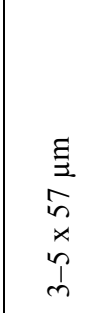 & 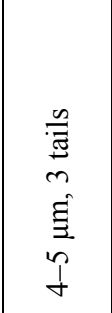 & 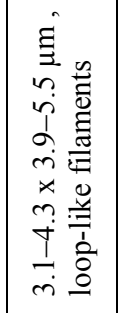 & 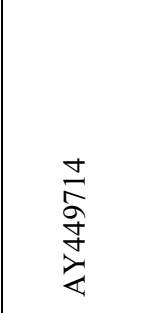 \\
\hline 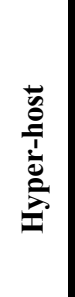 & 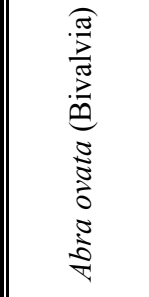 & 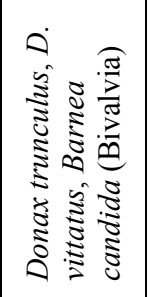 & 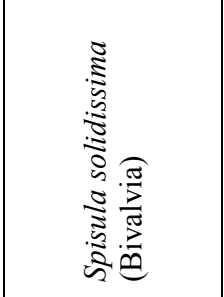 & 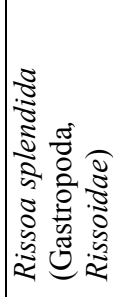 & 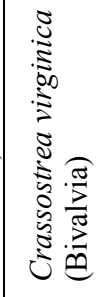 & 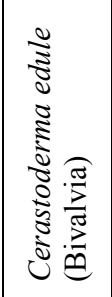 & 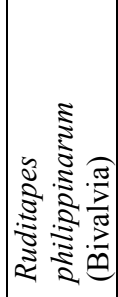 & 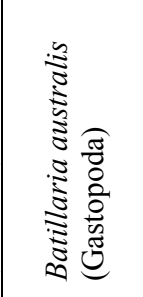 \\
\hline 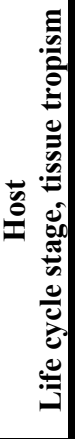 & 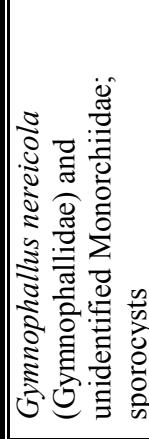 & 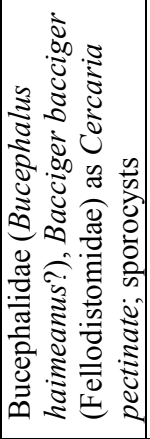 & 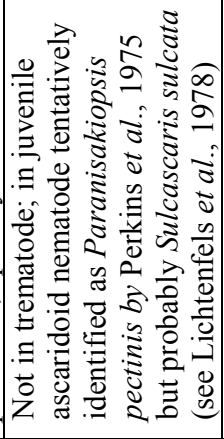 & 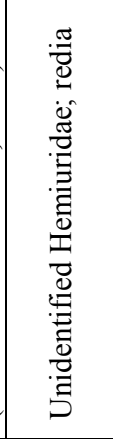 & 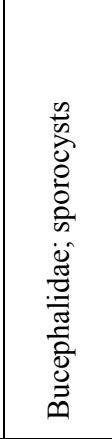 & 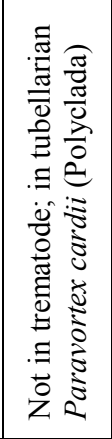 & 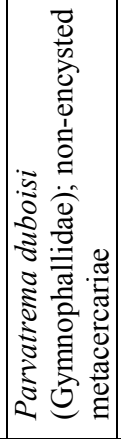 & 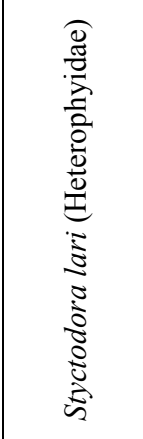 \\
\hline 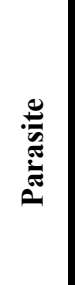 & 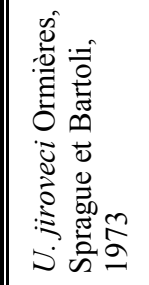 & 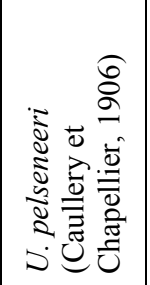 & 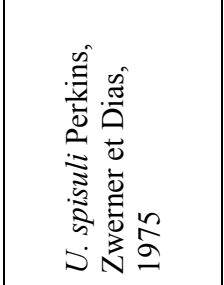 & 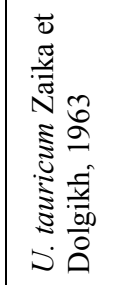 & 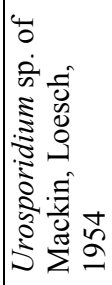 & 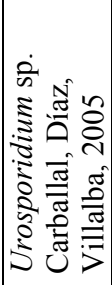 & 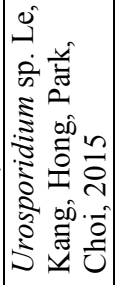 & 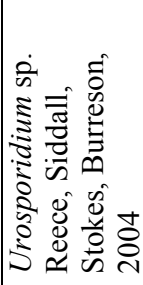 \\
\hline
\end{tabular}




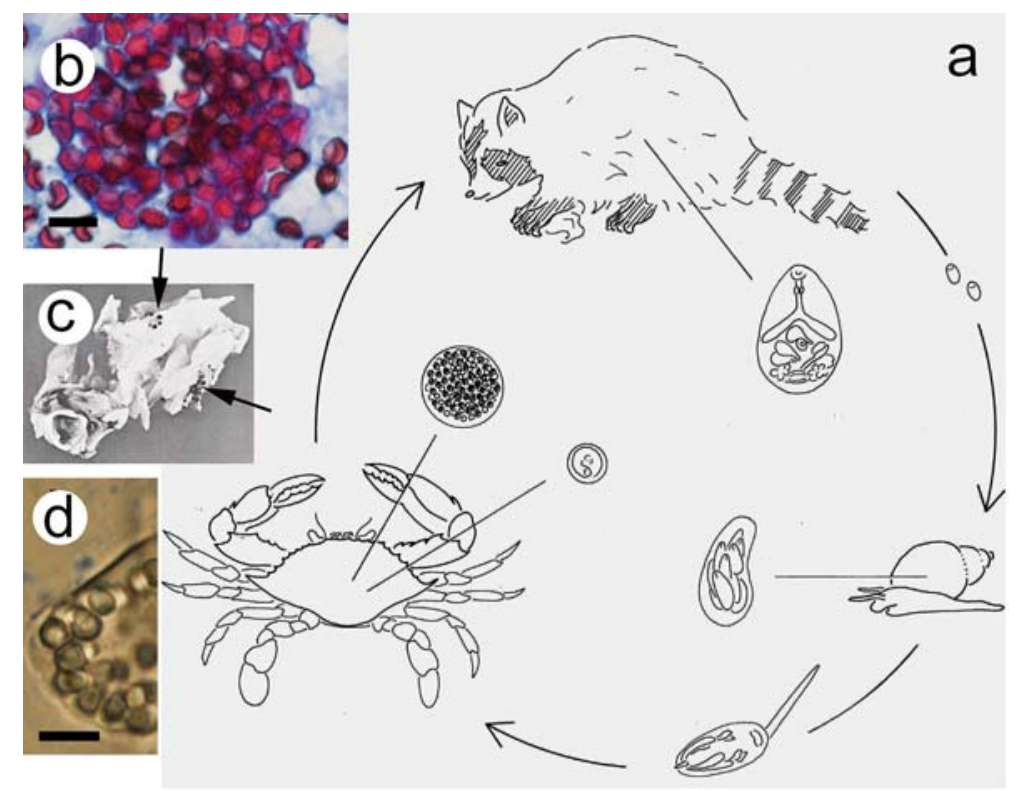

Fig. 4. Pepper spot disease in blue crabs caused by a Urosporidium crescens (Haplosporidia) infection in Microphallus basodactylophallus (Microphallidae).

a - life cycle of the digenean M. basodactylophallus. The adult worm in the intestine of the racoon discharges eggs in the host feces which are eaten by and hatch in at least six snail species serving as first intermediate hosts. From the enclosed larva, numerous swimming cercariae are produced asexually. These penetrate the blue crab and encyst as spherical metacercariae. When hyperparasitized by $U$. cresens, the encysted worm and its cyst wall enlarge to an easily visible size (from about $350 \mu \mathrm{m}$ to over $650 \mu \mathrm{m}$ in diameter), with brownish spores giving the heavily infected metacercaria a black appearance. Non hyperparasitized worms develop to adults if eaten by a proper bird or mammal final host (modified from Overstreet, 1978); b - spores from hyperparasitized metacercaria on smear of tissues of the crab with pepper disease. Haplosporidian spores stained red with Giemsa, scale bar $-5 \mu \mathrm{m}$; c - cooked crabmeat showing two areas with M. basodactylophallus metacercariae hyperparasitized with U. crescens ("pepper spots", arrows); $\mathrm{d}-$ live spores of $U$. crescens in the trematode, scale bar $-5 \mu \mathrm{m}$.

Рис. 4. Болезнь крабов "Реpper spot disease", вызванная заражением Urosporidium crescens (Haplosporidia) трематоды Microphallus basodactylophallus (Microphallidae).

a - жизненный цикл трематоды M. basodactylophallus. Взрослые черви в кишечнике енота откладывают яйца. Последние попадают в фекалии, на которых питается как минимум 6 видов улиток - первых промежуточных хозяев трематоды. Из личинки асексуальным путем формируются многочисленные свободно-плавающие церкарии. Церкарии проникают в краба, инцистируются и преобразуются в сферические метацеркарии. В случае гиперпаразитирования в них $U$. cresens, инцистированные трематоды увеличиваются в размере (с примерно $350 \mu \mathrm{m}$ до более чем $650 \mu \mathrm{m}$ в диаметре) и становятся заметны невооруженным говзом за счет коричневатых спор, которые придают метацеркариям темный цвет. Незараженные черви развиваются во взрослых особей при попадании в желудок окончательного хозяина, птицы или млекопитающнго (по Overstreet, 1978); b - споры из гиперпаразитированных метацеркарий на мазке тканей краба, зараженных "pepper disease". Споры гаплоспоридий окрашены красным с помощью окрашивания по Гимза-Романовскому, масштаб $-5 \mu \mathrm{m}$; c - вареное мясо краба с зонами заражения церкариями M. basodactylophallus c $U$. crescens ("pepper spots", стрелки); $\mathrm{d}$ - живые споры $U$. crescens в трематоде, масштаб $-5 \mu \mathrm{m}$.

an Malacobdella grossa on the pholadid bivalve Zirfaea crispate (see Jennings, Gibson, 1968). Urosporidium crescens reported from several hosts and from geographically disparate localities may not all be conspecific. The taxonomic problems should be alleviated once all or most of the species are sequenced.
A few species of Urosporidium can influence commercial sales of the hyper-hosts such as the surf clam with infected nematode and blue crab with pepper spot-buckshot (Fig. 4). In both cases, the spores are brownish but appear black to the naked eye. For example, consumers of the blue crab do not want to eat crabs with disease 
symptoms, such as the pepper spots. These spots are metacercariae that are packed with cysts and spores of $U$. crescens, which have caused the metacercariae and cyst wall to enlarge several times that of an uninfected metacercariae. As it turns out, if the consumer was to eat infected crabs raw, the uninfected metacercaria could cause a human infection with the trematode, but the hyperinfected metacercaria would not.

Pathological responses in cercariae and metacercariae cause mortality of the cercariae and castration of the metacercariae. This seems to be true for all species examined (Azevedo, Hine, 2017).

\section{Myxozoa}

The first myxosporidian described from a trematode was Fabespora vermicola (Fig. 5) infecting the apocreadiid Crassicutis archosar$g i$ in Mississippi (Overstreet, 1976b). The infection was rare and RMO has not seen it in $C$. archosargi since. Visual attempts made at the time of original collection to encounter it in the sparid fish host, Archosargus probatocephalus, to determine if both the fish and trematode contained the infection were also unsuccessful. However, in 1980, William Font, who was on sabbatical in RMO's laboratory, did find what was probably the same species of Fabespora (Table 3) in the cryptogonimid Metadena cf. spectanda in the sciaenid fishes Micropogonias undulatus and Cynoscion arenareus in Davis Bayou, Ocean Springs, Mississippi, from near where infected individuals of $A$. probatocephalus initially occurred (W. Font, R. Overstreet, unpublished). The trematode was identified as Metadena spectanda (Overstreet, 1971) from M. undulatus and another sciaenid fish, with the caveat that the species may differ from the larger Brazilian specimens used in the original description. This problem to determine if all trematode specimens from sciaenids in both South and North America are conspecific can now be solved with DNA sequencing. Sequencing could also solve the question as to whether all the myxosporidian (Fabespora) material from Mississippi is conspecific, relat-

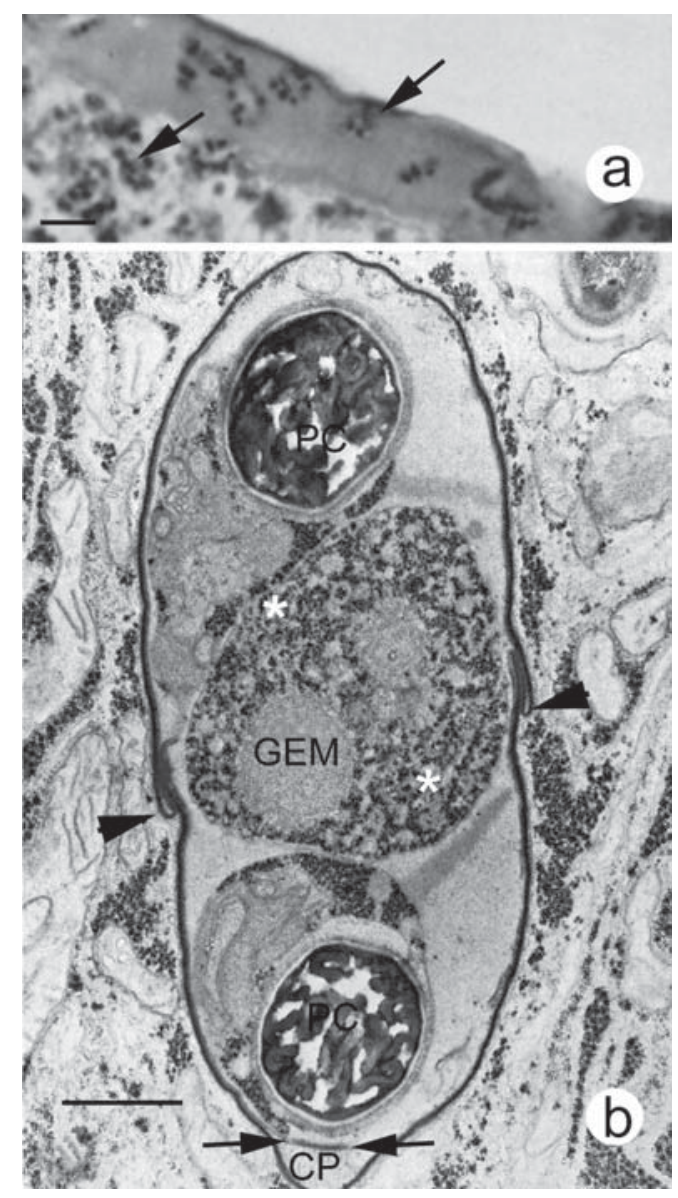

Fig. 5. Fabespora vermicola, the first myxosporidian species described from trematodes.

a $-F$. vermicola spores (arrows) in the tegument and parenchyma of the trematode Crassicutis archosargi (Apocreadiidae) collected from the sheepshead fish, Archosargus probatocephalus, scale bar - $20 \mu \mathrm{m} ; \mathrm{b}-$ ultrastructure of the $F$. vermicola sporoblast. Germinative cell (GEM) with rich glycogen pool (asterisks). CAP cell with mature polar capsules (PC). Note valvular sutures (arrowheads) separating polar cap (CP) from polar capsule (arrows), scale bar $-1 \mu \mathrm{m}$ (from Weidner, Overstreet, 1979, with permission form Springer-Verlag).

Рис. 5. Fabespora vermicola, первый вид миксоспоридий, описанный из трематод.

а - споры $F$. vermicola (стрелки) внутри тегумента трематоды Crassicutis archosargi (Apocreadiidae) из рыбы Archosargus probatocephalus, масштаб $20 \mu \mathrm{m} ; \mathrm{b}-$ ультраструктура споробласта $F$. vermicola. Герминальная клетка (GEM), богатая гранулами гликогена (звездочки); капсульная клетка со зрелыми полярными капсулами (РС). Вальвулярные швы указаны наконечниками стрелок. Видны структуры, разделяющие полярную шапочку СР от полярной капсулы (стрелки), масштаб $1 \mu \mathrm{m}$ (по Weidner, Overstreet, 1979, с разрешения издательства Springer-Verlag). 


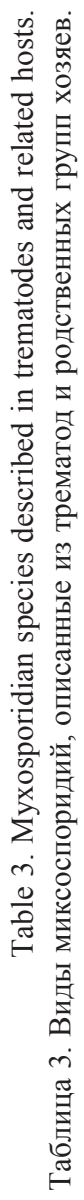

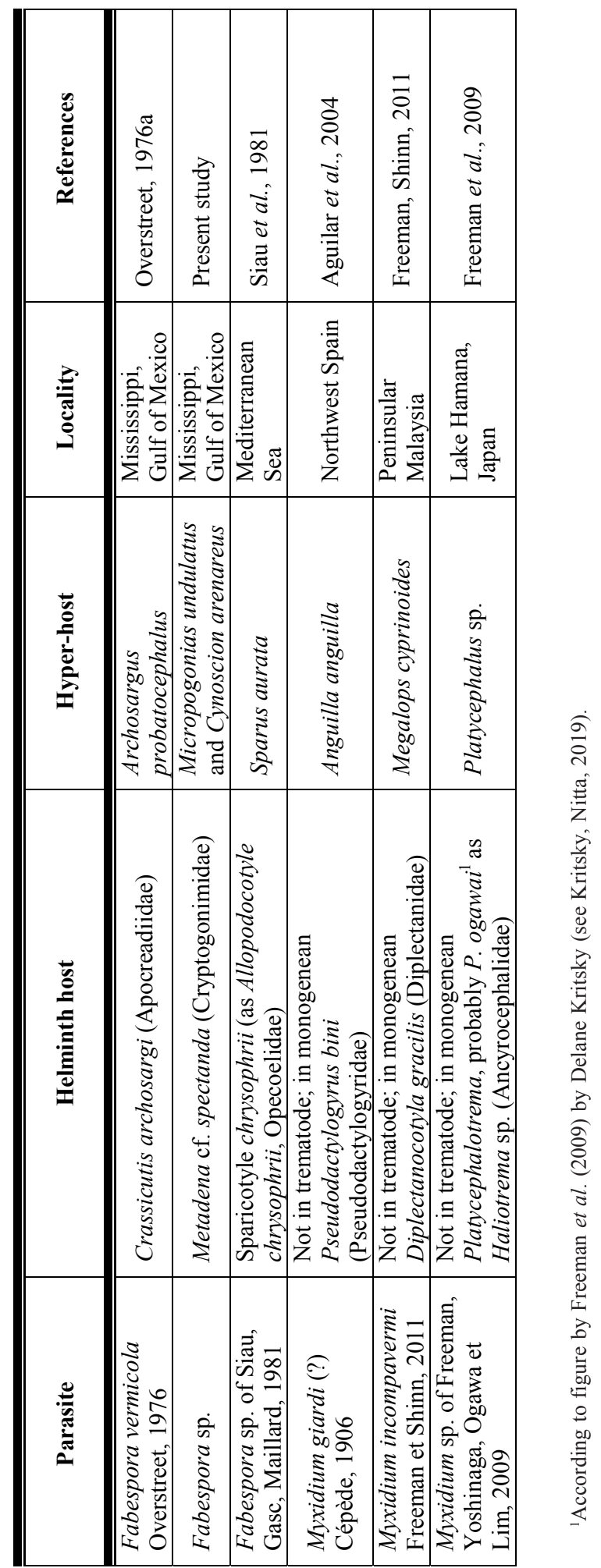


ed to another, and related to Fabespora spp. in fishes.

Another record of infection in a trematode was also identified as Fabespora sp., probably a different species (Siau et al., 1981). It occurred in the opecoelid Sparicotyle chrysophrii in Sparus aurata from the French Mediterranean Sea (Table 3), and the authors described ultrastructural aspects of sporogenesis as an earlier paper did (Weidner, Overstreet, 1979). The unusual presence of bundled microfilaments (identified as microtubules by Siau et al., 1981) in the extracellular space between the valvogenic and interior cells of the sporoblast attaching to the plasma membranes of both capsulogenic and germinative cells apparently allows the spores to propel themselves through host tissues by undulating their valves (Overstreet, 1976a; Weidner, Overstreet, 1979). Early developmental myxozoan stages occurred within and around trematode gonads; the mature disporous spores were enclosed in membrane and passed from the parenchyma to and through the tegument. Heavily infected specimens had necrotic gonads and a lack or paucity of eggs. In M. undulatus infections were found in 1 of 22 worms in the pyloric caeca and in 1 of 6 in the intestines, whereas in C. arenarius in 4 of 11 worms in the caeca and in 0 of 15 in the intestine, suggesting infections typically occur in young individual trematodes (W. Font, R. Overstreet, unpublished). Fabespora spp. also occur in fishes. The type-species, Fabespora nana, for which DNA has not been sequenced, infected the Knout goby, Mesogobius batrachocephalus, in the Black Sea (Naidenova, Zaika, 1969) as well as the gall bladder of the cuskeel Ophidion rochei but not M. batrachocephalus near Karadag in the Black Sea (Yurakhno, 2013). Unnamed species occurred in Merluccius hubbsi in the Argentine Sea (Cantatore et al., 2016) and in Centropomus undecimalis from Tampa Bay, Florida (Landsberg, 1993). Landsberg found the spores in the fish host's feces and questioned whether they had originally come from a trematode. The relationship between fish infections in the Black Sea and those from trematodes needs to be established.
Other platyhelminths also host myxosporidians (Table 3), such as Myxidium giardi being free (not encysted) in the ventral region near the opisthaptor of the monogenean Pseudodactylogyrus bini on the branchial tissues from 1 of 323 examined Anguilla anguilla but in none of the related monogenean on the same fish from northwestern Spain (Aguilar et al., 2004). Myxidium incomptavermi was reported from a gill-infesting monogenean (Diplectanocotyla gracilis) on Megalops cyprinoides in Malaysia (Freeman, Shinn, 2011). It was also detected by PCR in the kidney, spleen, and intestinal tract of the tarpon fish host. Sequences of 1,702 base pairs of SSU rDNA showed that $M$. incomptavermi occurred robustly at the base of the multivalvulidan clade (Kudoa, Sphaerospora, and Unicapsula) alongside with another species from Japan (Freeman et al., 2009; Table 3). These taxa were placed distantly from the unrelated freshwater and marine Myxidium clades. It is important to determine where Fabespora spp. from trematodes fit in that tree. All myxosporidian species infecting Platyhelminthes cause severe pathological alterations in the worm hosts.

\section{Conclusions}

Microsporidia in trematodes are common and are represented by several species. Trematode-infecting microsporidia with clarified phylogenetic position descend from the species infecting invertebrates (Unikaryion) and fish (Pleistophora and Ovipleistophora-like species). However phylogenetic position and origin of most microsporidia recovered from trematodes, remain unknown.

The representatives of only one Haplosporidia genus, Unikaryon, are known to infect trematodes, and this genus has a broad host range among marine parasitic and free-living marine invertebrates.

All trematode infections with myxoporidia were recovered from fish; they are caused by species belonging to different clades of Myxozoa, and have switched to trematodes from fish hosts. 
Parasitic "sporozoans" demonstrate diversity of characters and physiological mechanisms specific for each group, alongside with common adaptations in response to similar environmental conditions that have created morphologically alike forms from genetically dissimilar material. Indeed, the described above microscopic organisms exemplify an amazing power of convergent evolution, the phenomenon that A.A. Dobrovolskiy and other scholars of the Russian school of parasitology explicitly demonstrated on a variety of parasitic groups (Dogiel, 1962; Ginecynskaya, Dobrovolskii, 1978).

Acknowledgements. We thank Maryanne Anthony, Jean Jovonovich Alvillar, Joyce Shaw, and Janet Wright for their assistance with references. This paper was supported in part from a grant by BP Exploration \& Production, Inc, and Budgetary Program \#0124-2019-0005 at the Institute of Cytology RAS (YS).

The authors declare that there is no conflict of interest regarding the publication of this article. The authors are responsible for all data and figures presented in the paper.

\section{References}

Adl S.M., Simpson A.G., Lane C.E., Lukeš J., Bass D. et al. 2012. The revised classification of eukaryotes // J. Eukaryot. Microbiol. Vol.59. P.429-493.

Adl S.M., Bass D., Lane C.E., Lukeš,J., Schoch C.L. et al. 2019. Revisions to the classification, nomenclature, and diversity of eukaryotes // J. Eukaryot. Microbiol. Vol.66. P.4-119.

Aguilar A., Aragort W., Álvarez M.F., Leiro J.M., Sanmartín M. 2004. Hyperparasitism by Myxidium giardi Cépède 1906 (Myxozoa: Myxosporea) in Pseudodactylogyrus bini (Kikuchi, 1929) Gussev, 1965 (Monogenea: Dactylogyridae), a parasite of the European eel Anguilla anguilla L. // Bul. Eur. Ass. Fish Pathol. Vol.24. P.287-292.

Anderson T.J., Newman L.J., Lester R.J.G. 1993. Light and electron microscope study of Urosporidium cannoni $\mathrm{n}$. sp. haplosporidian parasite of the polyclad turbellarian Stylochus sp. // J. Eukaryot. Microbiol. Vol.40. P.162-168.

Azevedo C., Canning E.U. 1987. Ultrastructure of a microsporidian hyperparasite, Unikaryon legeri (Microsporida), of trematode larvae // J. Parasitol. Vol.73. P.214-223.

Azevedo C., Hine P.M. 2017. Haplosporidia // J. Archiba1d, A. Simpson, C. Slamovits (eds.). Handbook of the Protists. Springer, Cham. P.823-850.
Bojko J., Behringer D.C., Moler P. Reisinger L. 2020. Ovipleistophora diplostomuri, a parasite of fish and their trematodes, also infects the crayfish Procambarus bivittatus // J. Invert. Pathol. Vol.169. doi: 10.1016/j.jip.2019.107306 (Ahead of print).

Brumpt E. 1922. Précis de parasitologie. $3^{\text {rd }}$ ed. Paris: Masson et Cie. xv +1216 p.

Burreson E.M., Ford S.E. 2004. A review of recent information on the Haplosporidia, with special reference to Haplosporidium nelsoni (MSX disease) // Aquat. Living Resour. Vol.17. P.499-517.

Canning E.U. 1972. Nosema eurytremae, a replacement name for the secondary homonym Perezia helminthorum Canning and Basch, a parasite of digenean larvae // J. Invertebr. Pathol. Vol.20. P.371.

Canning E.U. 1975. The microsporidian parasites of Platyhelminthes: their morphology, development, transmission and pathogenicity // Commonwealth Institute of Helminthology Miscellaneous Publications No.2. P.1-32.

Canning E.U., Barker R.J., Hammond J.C., Nicholas J.P. 1983. Unikaryon slaptonleyi sp. nov. (Microspora: Unikaryonidae) isolated from echinostome and strigeid larvae from Lymnaea peregra: observations on its morphology, transmission and pathogenicity // Parasitology Vol.87. P.175-184.

Canning E.U., Basch P.F. 1968. Perezia helminthorum sp. nov., a microsporidian hyperparasite of trematode larvae from Malaysian snails // Parasitology. Vol.58. P.341-347.

Canning E.U., Gunn A. 1984. Nosema helminthorum Moniez, 1887 (Microspora, Nosematidae): a taxonomic enigma 1 // J. Protozool. Vol.31. P. 525-531.

Canning E.U., Lai P.K., Lie K.J. 1974. Microsporidian parasites of trematode larvae from aquatic snails in West Malaysia // J. Protozool. Vol.21. P.19-25.

Canning E.U., Madhavi R. 1977. Studies on two new species of Microsporidia hyperparasitic in adult Allocreadium fasciatusi (Trematoda, Allocreadiidae) // Parasitology. Vol.75. P.293-300.

Canning E.U., Nicholas J.P. 1974. Light and electron microscope observations on Unikaryon legeri (Microsporida, Nosematidae), a parasite of the metacercaria of Meigymnophallus minutus in Cardium edule // J. Invert. Pathol. Vol.23. P.92-100.

Canning E.U., Olson A.C. 1980. Nosema lepocreadii sp. n., a parasite of Lepocreadium manteri (Digenea: Lepocreadiidae) from the gut of the California grunion, Leuresthes tenuis // J. Parasitol. Vol.66. P.154159.

Cantatore D.M.P., Irigoitia M.M., Holzer A.S., Timi J.T. 2016. Myxozoans as biological tags for stock identification of the Argentine hake, Merluccius hubbsi (Gadiformes: Merlucciidae) // Parasitology Vol.143. P.732-740.

Carballal M.J., Díaz S., Villalba A. 2005. Urosporidium sp. hyperparasite of the turbellarian Paravortex cardii in the cockle Cerastoderma edule // J. Invertebr. Pathol. Vol.90. P.104-107.

Caullery M., Mesnil F. 1905. Sur quelques nouvelles Haplosporidies d'Annélides // C. R. Soc. Biol. T.58. P.580-583.

Caullery M., Chappellier A. 1906. Anurosporidium pelseneeri, n. g. n. sp. haplosporidie infectant les sporo- 
cysts d'un trématode parasite du Donax trunculus L. // C. R. Soc. Biol, T.60. P.325-328.

Colley F.C. 1975. Nosema eurytremae Canning 1972 in heart tissue of the land snail Bradybaena similaris // Southeast Asian J. Trop. Med. Public Health. Vol.6. P.142-143.

Colley F.C., Joe L.K., Zaman V., Canning E.U. 1975. Light and electron microscopical study of Nosema eurytremae // J. Invertebr. Pathol. Vol.26. P.11-20.

Couch J.A. 1974. Pathological effects of Urosporidium (Haplosporida) infection in microphallid metacercariae // J. Invertebr. Pathol. Vol.23. P.389-396.

De Morgan A. 1915. Are Atoms Worlds?: Siphonaptera // D.E. Smith (ed.). A Budget of Paradoxes. II (2nd ed.). P.191.

De Turk W.E. 1940.The occurrence and development of a hyperparasite, Urosporidium crescens sp. nov. (Sporozoa, Haplosporidia), which infests the metacercariae of Spelotrema nicolli, parasitic in Callinectes sapidus // J. Elisha Mitchell Sci. Soc. Vol.56. P.231-232.

Diamant A., Paperna I. 1985. The development and ultrastructure of Nosema ceratomyxae sp. nov., a microsporidian hyperparasite of the myxosporean Ceratomyxa sp. from Red Sea rabbitfish (Siganidae) // Protistologica. Vol.21. P.249-258.

Dissanaike A.S. 1957. On Protozoa hyper-parasitic in helminths, with some observations on Nosema helminthorum Moniez, 1887 // J. Helminthol. Vol.31. P.47-64.

Dogiel V.A. 1962. [General Parasitology]. Leningrad: Leningrad University Press. 464 p. [In Russian]

Dollfus R.P. 1912. Contribution à l'étude des trématodes marins des côtes du Boulonnais: Une métacercaire margaritigène parasite de Donax vittatus da Costa // Mém. Soc. Zool. France. Vol.25. P.85-144.

Dollfus R.P. 1925. Liste critique des cercaires marines à queue sétigère signalées jusqu'à present // Trav. Sta. Zool. Wimereux. Vol.9. P.43-65.

Dollfus R.P. 1941. Titres et travaux scientifiques de Robert-Ph. Dollfus // In-4 Paris Octobre 1941 Imprimerie Barnéoud-Laval. 76 p.

Dollfus R.P. 1944. Hyperparasitisme et castration parasitaire par un sporozoaire chez un Cestode // C. R. Acad. Sc. T.216. No.11 (1943). P.270-272, fig. 1.

Dollfus R.P. 1946. Parasites (animaux et végétaux) des Helminthes: Hyperparasites, ennemis et predateurs des Helminthes parasites et des Helminthes libres // Encycl. Biol. 37. Paris: Lechevalier. 481 p.

Franzen C., Nassonova E.S., Schölmerich J., Issi I.V. 2006. Transfer of the members of the genus Brachiola (microsporidia) to the genus Anncaliia based on ultrastructural and molecular data // J. Eukaryot. Microbiol. Vol.53. P.26-35.

Freeman M.A., Bell A.S., Sommerville C. 2003. A hyperparasitic microsporidian infecting the salmon louse, Lepeophtheirus salmonis: an rDNA-based molecular phylogenetic study // J. Fish Dis. Vol. 26. P.667-676.

Freeman M.A., Sommerville C. 2011. Original observations of Desmozoon lepeophtherii, a microsporidian hyperparasite infecting the salmon louse Lepeophtheirus salmonis, and its subsequent detection by other researchers // Parasites Vectors Vol.4: e231. http:// dx.doi.org/10.1186/1756-3305-4-231.

Freeman M.A., Shinn A.P. 2011. Myxosporean hyperparasites of gill monogeneans are basal to the Multival- vulida // Parasites \& Vectors. Vol.4. P.220.

Freeman M.A., Yoshinaga T., Ogawa K., Lim L.H.S. 2009. Myxidium-like myxosporean hyperparasites of gill monogeneans are basal multivalvulidans // Proc 14th EAFP Meeting, Prague. 273 p.

Ginetsinskaya T.A. 1968. [Trematodes, their life cycles, biology and evolution]. Leningrad: Nauka. 411 p. [In Russian]

Ginetsinskaya T.A., Dobrovolskii A.A. 1978. [Special parasitology. Vol.2. Parasitic worms, Mollusca and Arthropoda]. Moscow: Vysshaya Shkola. 292 p. [In Russian]

Guyénot E. 1943. Sur un Haplosporidie, parasite dans un sporocyste de la Pholade, Barnea candida L. // Rev. suisse Zool. T.50. P.283-286.

Guyénot E., Naville A. 1924. Glugea encyclometrae n. sp. et G. ghigii n. sp. parasites de platodes et leur développement dans l'hôte vertébré ("Tropidonotus natrix L.”) // Rev. suisse Zool. T.31. P.75-115.

Guyénot E., Naville A., Ponse K. 1925. Deux microsporidies parasites de trématodes // Rev. suisse Zool. T.31. P.399-421.

Higby G.C., Canning E.U., Pilley B.M., Bush P.J. 1979. Propagation of Nosema eurytremae (Microsporida: Nosematidae) from trematode larvae, in abnormal hosts and in tissue culture // Parasitology. Vol.78. P.155-170.

Howell M. 1967. The trematode, Bucephalis longicornutus (Manter, 1954) in the New Zealand mud-oyster, Ostrea lutaria // Trans. Roy. Soc. N. Z., Zool. Vol.8. P.221-237.

Hunninen A.V., Wichterman R. 1938. Hyperparasitism: a species of Hexamita (Protozoa, Mastigophora) found in the reproductive systems of Deropristis inflata (Trematoda) from marine eels // J. Parasitol. Vol.24. P.95-101.

Hussey K.L. 1971. A Microsporidian hyperparasite of strigeoid trematodes, Nosema strigeoideae sp. n. // J. Protozoology. Vol.18. P.676-679.

Hussey K.L. 1973. Effects of microsporidian infection on larval trematodes: infection with Nosema strigeodeae or N. echinostomi // J. Invertebr. Pathol. Vol.22. P.193-198.

Jennings J.B., Gibson R. 1968. The structure and life history of Haplosporidium malacobdellae sp. nov., a new sporozoan from the entocommensal rhynchocoelan Malacobdella grossa (O.F. Müller) // Arch. Protistenk. Bd.111. P.31-37.

Kritsky D.C., Nitta M. 2019. Dactylogyrids (Platyhelminthes: Monogenoidea) infecting the gill lamellae of Flatheads (Scorpaeniformes: Platycephalidae), with proposal of Platycephalotrema n. gen. and descriptions of new species from Australia and Japan // Diversity. Vol.11. P.132. doi:10.3390/d11080132.

Kudo R. 1924. A Biologic and taxonomic study of the microsporidia // Illinois Biol. Monogr. Vol.9. P.1268.

Kudo R.R. 1939. Observations on Nosema notabilis n. sp. parasitic in a myxosporidian // Anat. Record (Suppl.). Vol.75. P.153.

Lai P.F., Canning E.U. 1980. Infectivity of a microsporidium of mosquitoes (Nosema algerae) to larval stages of Schistosoma mansoni in Biomphalaria glabrata // Int. J. Parasitol. Vol.10. P.293-301. 
Landsberg J.H. 1993. Myxosporean parasites of common snook in Florida // J. Aquat. Anim. Health. Vol.5. P.102-109.

Le T.C., Kang H.-S., Hong H.-K., Park K.-J., Choi K.-S. 2015. First report of Urosporidium sp., a haplosporidian hyperparasite infecting digenean trematode Parvatrema duboisi in Manila clam, Ruditapes philippinarium on the west coast of Korea // J. Invert. Pathol. Vol.130. P.141-146.

Levron C., Ternengo S., Toguebaye B.S., Marchand B. 2004. Ultrastructural description of the life cycle of Nosema diphterostomi sp. n., a microsporidia hyperparasite of Diphterostomum brusinae (Digenea: Zoogonidae), intestinal parasite of Diplodus annularis (Pisces: Teleostei) // Acta Protozool. Vol.43. P.329336.

Levron C., Ternengo S., Toguebaye B.S., Marchand B. 2005. Ultrastructural description of the life cycle of Nosema monorchis n. sp.(Microspora, Nosematidae), hyperparasite of Monorchis parvus (Digenea, Monorchiidae), intestinal parasite of Diplodus annularis (Pisces, Teleostei) // Eur. J. Protistol. Vol.41. P.251-256.

Lichtenfels J.R., Bier J.W., Madden P.A. 1978. Larval anisakid (Sulcascaris) nematodes from Atlantic molluscs with marine turtles as definitive hosts // Trans. Amer. Micros. Soc. Vol.97. P.199-207.

Lie K.J., Basch P.F., Umathevy T. 1966. Studies on Echinostomatidae (Trematoda) in Malaya. XII. Antagonism between two species of echinostome trematodes in the same lymnaeid snail // J. Parasitol. Vol.52. P.454-457.

Lie K.J., Basch P.F. 1970. Occurrence of Perezia helminthorum Canning and Basch, 1968 (Microsporida: Nosematidae) in trematode larvae of fresh-water snails // SE Asian J. Trop. Med. Pub. Hlth. Vol.1. P.419-420.

Lovy J., Friend S.E. 2017. Phylogeny and morphology of Ovipleistophora diplostomuri n. sp. (Microsporidia) with a unique dual-host tropism for bluegill sunfish and the digenean parasite Posthodiplostomum minimum (Strigeatida) // Parasitology. Vol.144. P.1898-1911.

Lutz A., Splendore A. 1908. Ueber Pebrine und verwandte mikrosporidien. Zweite Mitteilung // Centralbl. Bakteriol. Parasitenkd. Infektionskr. Hyg. Abt.I. Bd.46. S.311-315.

Mabbott N.A. 2018. The influence of parasite infections on host immunity to co-infection with other pathogens // Frontiers in Immunology. Vol.9. P.2579.

Mackin J.G., Loesch H. 1954. A haplosporidian hyperparasite of oysters // Proc. Natl. Shellf. Assoc. Vol.45. P.182-183.

Martin W.E. 1936. A sporozoan parasite of larval trematodes // J. Parasitol. Vol.22. P.536.

Menke J.H. 1968. Urosporidium astomatum n. sp., a haplosporidian infecting the metacercariae of Parvatrema donacis, a trematode parasite of the bivalve Donax variabilis. Master's Thesis. Texas A\&M University. 40 p. (plus i-ix).

Morris D.J., Freeman M.A. 2010. Hyperparasitism has wide-ranging implications for studies on the invertebrate phase of myxosporean (Myxozoa) life cycles // Int. J. Parasitol. Vol.40. P.357-369.

Naidenova N.N., Zaika W.E. 1969. [Two new species of Protozoa from the fishes of the Black Sea] // Parazitologiia (Leningrad). Vol.3. P.97-101 [in Russian, with English summary].
Nylund S., Nylund A., Watanabe K., Arnesen C.E., Karlsbakk E. 2010. Paranucleospora theridion n. gen., n. sp. (Microsporidia, Enterocytozoonidae) with a life cycle in the salmon louse (Lepeophtheirus salmonis, Copepoda) and Atlantic salmon (Salmo salar) // J. Eukaryot. Microbiol. Vol.57. P.95-114.

Ormières R., Sprague V., Bartoli P. 1973. Light and electron microscope study of a new species of Urosporidium (Haplosporida), hyperparasite of trematode sporocysts in the clam Abra ovata // J. Invertebr. Pathol. Vol.21. P.71-86.

Overstreet R.M. 1971. Metadena spectanda Travassos, Freitas, and Bührnheim, 1967 (Digenea: Cryptogonimidae) in estuarine fishes from the Gulf of Mexico // Proc. Helm. Soc. Wash. Vol.38. P.156-158.

Overstreet R.M. 1976a. Fabespora vermicola sp. n., the first myxosporidan from a platyhelminth // J. Parasitol. Vol.62. P.680-684.

Overstreet R.M. 1976b. A redescription of Crassicutis archosargi, a digenean exhibiting an unusual tegumental attachment // J. Parasitol. Vol.62. P.702-708.

Overstreet R.M. 1978. Marine Maladies? Worms, Germs, and Other Symbionts from the Northern Gulf of Mexico // Mississippi-Alabama Sea Grant Consortium, MASGP-78-021. 140 p.

Overstreet R.M., Lotz J.M. 2016. Host-symbiont relationships: understanding the change from guest to pest // C.J. Hurst (ed.). The Rasputin Effect: when Commensals and Symbionts Become Parasitic. Advances in Environmental Microbiology Vol.3. P.27-64.

Palmieri J.R., Cali A., Heckmann R.A. 1976. Experimental biological control of the eye fluke, Diplostomum spathaceum, by a protozoan hyperparasite, Nosema strigeoidae (Protozoa: Microsporida) // J. Parasitol. Vol.62. P.325-326.

Palmieri J.R., Lai P.C., Sullivan J.T., Cali A. 1978. Effects of Microsporida on snail and trematode tissue // SE Asian J. Trop. Med. Pub. Hlth. Vol.9. P.256-259.

Paperna I., Sabnai I., Castel M. 1978. Microsporidian infection in the cyst wall of trematode metacercariae encysted in fish // Ann. Parasitol. Hum. Comp. T.53. P. 123-130.

Pekkarinen M., Lom J., Nilsen F. 2002. Ovipleistophora gen. n., a new genus for Pleistophora mirandellaelike microsporidia// Dis. Aquat. Organ. Vol.48. P.133142.

Perkins F.O., Zwerner D.E., Dies R.K. 1975. The hyperparasite, Urosporidium spisuli sp. n. (Haplosporea), and its effects on the surf clam industry // J. Parasitol. Vol.61. P.944-949.

Pilley B.M., Canning E.U., Hammond J.C. 1978. The use of a microinjection procedure for large-scale production of the microsporidian Nosema eurytremae in Pieris brassicae // J. Invertebr. Pathol. Vol.32. P.355358.

Poddubnaya L.G., Tokarev Y.S., Issi I.V. 2006. A new microsporidium Paratuzetia kupermani gen. et sp. n.(Microsporidia), a hyperparasite of the procercoid of the cestode Khawia armeniaca Chol. 1915 (Cestoda, Caryophyllidea) // Protistology. Vol.4. P.269-277.

Reece K.S., Siddall M.E., Stokes N.A., Burreson E.M. 2004. Molecular phylogeny of the Haplosporidia based on two independent gene sequences // J. Parasitol. Vol.90. P.1111-1122. 
Sanders J.L., Lawrence C., Nichols D.K., Brubaker J.F., Peterson T.S., Murray K.N., Kent M.L. 2010. Pleistophora hyphessobryconis (Microsporidia) infecting zebrafish Danio rerio in research facilities // Dis. Aquat. Organ. Vol.91. P.47-56.

Schäller G. 1959. Microsporidienbefall und Degenerationserscheinungen der Trematodenlarven im Zwischenwirt (Tropidiscus planorbis) // Z. Wiss. Zool. Bd.162. S.144-190.

Sene A., Ba C.T., Marchan B., Toguebaye B.S. 1997. Ultrastructure of Unikaryon nomimoscolexi n. sp. (Microsporida, Unikaryonidae), a parasite of Nomimoscolex sp. (Cestoda, Proteocephalidea) from the gut of Clarotes laticeps (Pisces, Teleostei, Bagridae) // Dis. Aquat. Organ. Vol.29. P.35-40.

Shields J.D., Overstreet R.M. 2007. Diseases, parasites, and other symbionts. Chapter 8 // V.S. Kennedy, L.E. Cronin (eds.). The Blue Crab, Callinectes sapidus. College Park, Maryland Sea Grant. P.299-417.

Shigina N.G. 1986. [Microsporidia species of trematodes, their biology and possible use for biological control] // Protozoologiya. No.10 (Microsporidia). Leningrad: Nauka. P.167-182 [in Russian with English summary].

Shigina N.G., Grobov O.F. 1972. [Nosema diplostomi sp. n. (Microsporidia: Nosematidae), a hyperparasite of trematodes of the genus Diplostomum] // Parazitologiya. Vol.6. P.469-475 [in Russian with English summary].

Siau Y., Gasc C., Maillard C. 1981. Premières observations ultrastructurales d'une myxosporide appartenant au genre Fabespora, parasite de trématode // Protistologia. T.17. P.131-137.

Smith R.J. 1959. Ancylid snails: First intermediate host to certain trematodes with notes on ancylids as a new host for Megalodiscus and Haematoloechus // Tr. Am. Micr. Soc. Vol.78. P.228-231.

Sokolova Y.Y., Paskerova G.G., Rotari Y.M., Nassonova E.S., Smirnov A.V. 2013. Fine structure of Metchnikovella incurvata Caullery and Mesnil 1914 (microsporidia), a hyperparasite of gregarines Polyrhabdina sp. from the polychaete Pygospio elegans // Parasitology. Vol.140. P.855-867.

Sokolova Y.Y., Paskerova G.G., Rotari Y.M., Nassonova E.S., Smirnov A.V. 2014. Description of Metchnikovella spiralis $\mathrm{sp}$. n. (Microsporidia: Metchnikovellidae), with notes on the ultrastructure of metchnikovellids // Parasitology. Vol.141. P.1108-1122.

Sokolova Y.Y., Overstreet R.M. 2018. A new microsporidium, Apotaspora heleios n. g., n. sp. from the Riverine grass shrimp Palaemonetes paludosus (Decapoda: Caridea: Palaemonidae) // J. Invert. Pathol. Vol.157. P.125-135.

Sokolova Y., Weidner E., DeMario P. 2020. Development of Anncaliia algerae (Microsporidia) in Drosophila melanogaster // J. Eukaryot. Microbiol. Vol. 67. P.125-131.

Sprague V. 1954. Protozoa // P.S. Galtsoff (ed.). Gulf of Mexico - its origin, waters and marine life. U.S. Fish and Wildlife Serv. Fish. Bull. Vol.55. P.243-256.

Sprague V. 1964. Nosema dollfusi n. sp. (Microsporidia, Nosematidae), a hyperparasite of Bucephalus cuculus in Crassostrea virginica // J. Protozool. Vol.11. P.381385.

Sprague V. 1970. Some protozoan parasites and hyperparasites in marine bivalve mollusks // S.F. Sneiszko (ed.). A Symposium on Diseases of Fishes and Shell- fishes, Spec. Publ. 5. Washington, D.C.: American Fisheries Society. P.511-526.

Sprague V. 1977. Annotated list of species of Microsporidia // L.A. Bulla Jr., T.C. Cheng (eds.). Comparative Pathobiology. Vol.2, Systematics of the Microsporidia. New York: Plenum Press. P.31-334.

Stentiford G.D., Feist S.W., Stone D.M., Bateman K.S., Dunn A.M. 2013. Microsporidia: diverse, dynamic, and emergent pathogens in aquatic systems // Trends Parasitol. Vol.29. P.567-578.

Stentiford G.D., Ramilo A., Abollo E., Kerr R., Bateman K.S., Feist S.W., Bass D., Villalba A. 2017a. Hyperspora aquatica n. gn., n. sp. (Microsporidia), hyperparasitic in Marteilia cochillia (Paramyxida), is closely related to crustacean-infecting microspordian taxa // Parasitology. Vol.144. P.186-199.

Stentiford G.D., Ross S., Minardi D., Feist S., Bateman K., Gainey P., Troman C., Bass D. 2017b. Evidence for trophic transfer of Inodosporus octospora and Ovipleistophora arlo n. sp. (Microsporidia) between crustacean and ?sh hosts.// Parasitology. Vol.145. P.1105-1117.

Summerfelt R.C., Goodwin A.E. 2010. Ovipleistophoriasis: A Microsporidian Disease of the Golden Shiner Ovary / / AFS-FHS (American Fisheries Society Fish Health Section). FHS blue book: suggested procedures for the detection and identification of certain finfish and shellfish pathogens, 2010 edition. American Fisheries Society - Fish Health Section, Bethesda, Maryland, USA.

Swift J. 1910 (1733). On Poetry: a Rhapsody // E.W. Browning (ed.). The Poems of Jonathan Swift, D.D., Volume I. London: G. Bell and Sons, Ltd.

Toguebaye B.S., Quilichini Y., Diagne P.M., Marchand B. 2014. Ultrastructure and development of Nosema podocotyloidis $\mathrm{n}$. sp. (Microsporidia), a hyperparasite of Podocotyloides magnatestis (Trematoda), a parasite of Parapristipoma octolineatum (Teleostei) // Parasite. Vol.21. P.44. doi: 10.1051/parasite/2014044.

Vavra J., Undeen A.H. 1970. Nosema algerae n. sp.(Cnidospora, Microsporida) a pathogen in a laboratory colony of Anopheles stephensi Liston (Diptera, Culicidae) // J. Protozool. Vol.17. P.240-249.

Voronin V.N. 1974. [On parasitism of microsporidians (Microsporida, Nosematidae) in parthogenetic generations and cercariae of trematodes from freshwater molluscs] // Parazitologiya (Leningrad). Vol.8. P.359364 [in Russian].

Vossbrinck C.R., Debrunner-Vossbrinck B.A., Weiss L.M. 2014. Phylogeny of the Microsporidia // L.M. Weiss, J.J. Becnel (eds.). Microsporidia: pathogens of opportunity: First Edition. Wiley-Blackwell. P.203-220.

Weidner E., Overstreet R.M. 1979. Sporogenesis of a myxosporidan with motile spores // Cell Tissue Res. Vol.201. P.331-342.

Yurakhno V.M. 2013. The nature protection aspect of the Black Sea fish myxosporean studies // Vestn. Zool. Vol.47. P.62-70.

Zaika V.E., Dolgikh A.V. 1963. [A rare case of haplosporidian hyperparasitism by Urosporidium tauricum $\mathrm{sp}$. $\mathrm{n}$. in partenites of trematodes of the family Hemiuridae Lühe from the mollusk Rissoa splendida Eichw] // Zool. Zhurn. Vol.42. P.1727-1729 [in Russian, with English summary].

Responsible editor E.N. Temereva 